\title{
Spectrum of $p$-wave hidden-charm exotic mesons in the diquark model
}

\author{
Jesse F. Giron $\odot^{*}$ and Richard F. Lebed $\odot^{\dagger}$ \\ Department of Physics, Arizona State University, Tempe, Arizona 85287, USA
}

(Received 12 March 2020; accepted 13 April 2020; published 27 April 2020)

\begin{abstract}
We study the fine structure in the spectrum of known and predicted negative-parity hidden-charm exotic meson states, which comprise the lowest $P$-wave multiplet in the dynamical diquark model. Starting with a form previously shown to successfully describe the $S$-wave states, we develop a 5-parameter Hamiltonian that includes spin-orbit and tensor terms. After discussing the experimental status of the observed $J^{P C}=$ $1^{--}$states $Y$ with respect to masses and decay modes (classified by eigenvalues of heavy-quark spin), we note a number of inconsistencies between measurements from different experiments that complicate a unique determination of the spectrum. Outlining a variety of scenarios for interpreting the $Y$ data, we perform fits to each one, obtaining results that demonstrate differing possibilities for the $P$-wave spectra. Choosing one of these fits for illustration, we predict masses for all 28 isomultiplets in this $1 P$ multiplet, compare the results to tantalizing hints in the data, and discuss the rich discovery potential for new states.
\end{abstract}

DOI: 10.1103/PhysRevD.101.074032

\section{INTRODUCTION}

Although over 40 heavy-quark hidden-flavor exotic hadron candidates have now been observed by various experiments in the past two decades, no single theoretical picture to describe their structure has yet emerged as the obvious favorite. Several of the states [e.g., $X(3872)]$ lie provocatively close to 2 -hadron thresholds and suggest hadronic molecules or threshold effects, but others do not. Some prefer to decay only to conventional quarkonium states of one particular heavy-quark spin eigenvalue (e.g., $\psi$ rather than $h_{c}$ ), hinting at particular quarkonium cores (as in the hadroquarkonium model), but others [ e.g., $Z_{b}(10610) \rightarrow \Upsilon, h_{b}$ ] mix heavy-quark spin eigenstates. Some neutral exotics [e.g., Y(4260)] match the properties expected for hybrid states. And some [e.g., $\left.Z_{c}(4430)\right]$ have thus far only been clearly seen to decay to an excited quarkonium state $[\psi(2 S)]$ even though the ground state $(J / \psi)$ is available, suggesting a large spatial extent for the exotic hadron wave function, which is a feature of some diquark models. The current experimental status of the exotic hadron candidates, as well as detailed descriptions of the aforementioned theoretical pictures, are presented in a number of recent reviews [1-11].

\footnotetext{
jfgiron@asu.edu

Richard.Lebed@asu.edu
}

Published by the American Physical Society under the terms of the Creative Commons Attribution 4.0 International license. Further distribution of this work must maintain attribution to the author(s) and the published article's title, journal citation, and DOI. Funded by SCOAP .
In this paper we continue the development of the dynamical diquark model, specifically by studying the spectroscopy of exotic hidden-charm mesons of negative parity, which lie several hundred $\mathrm{MeV}$ above the lowest (positive-parity) states. The dynamical diquark model is based upon a physical picture [12] in which 4-quark states can be clearly identified as having a diquark $\delta$ [in the color$\overline{\mathbf{3}}$ combination $\left.(Q q)_{\overline{\mathbf{3}}}\right]$-antidiquark $\bar{\delta}\left[(\bar{Q} \bar{q})_{3}\right]$ structure only if the $\delta$ - $\bar{\delta}$ pair can achieve sufficient spatial separation as to evade instantaneous recombination into 2-meson pairs $(Q \bar{q})-(\bar{Q} q)$ or $(Q \bar{Q})-(q \bar{q})$. The heavy-quark pair $Q \bar{Q}$ in this paper is exclusively $c \bar{c}$, although $b \bar{b}$ states can also be analyzed this way, as well as $b \bar{c}$ states (if the full state's discrete quantum numbers are handled carefully [13]), and also pentaquark states [14] by using successive colortriplet attractions to form a triquark $\bar{\theta} \equiv\left[\bar{Q}\left(q_{1} q_{2}\right)_{\overline{\mathbf{3}}}\right]_{\mathbf{3}}$ quasiparticle, which can then form pentaquark states via the combination $\bar{\theta} \delta$. Alternate constructions of triquarkdiquark pentaquarks are studied in Refs. [15,16].

This initial picture has been developed in stages into the dynamical diquark model. First, the nature of the state as a well-separated $\delta-\bar{\delta}$ pair suggests a color flux tube connecting the pair, meaning that the most natural formalism for describing their spectroscopy uses multiplets of the BornOppenheimer (BO) approximation, the same way that hybrid mesons have been treated for decades [17]. The spectroscopy for $\delta-\bar{\delta}$ (and $\bar{\theta}-\delta$ ) states is presented in Ref. [13] (including detailed definitions of the standard BO potential nomenclature, e.g., $\Sigma_{u}^{-}$, etc.), and the most relevant expressions are reprised in Sec. II below; one key result informing this work is that the lowest $\left[\Sigma_{g}^{+}(1 S)\right]$ multiplet consists entirely of positive-parity states, while 
the first excited multiplet $\left[\Sigma_{g}^{+}(1 P)\right]$ contains only negativeparity states, a fact mentioned above.

The next step in building the model is to obtain explicit forms for the BO potentials provided by the flux tube, which is accomplished by numerically computing (primarily) gluonic potentials between static heavy quarks on the lattice, as in Refs. [18-22]. These potentials are then fed into coupled Schrödinger equations, which are numerically solved to obtain the mass eigenvalues for the $\delta-\bar{\delta}$ states. The results of these calculations [23] represent the spin- and isospin-averaged masses for each multiplet, with the lowest few being $1 S, 1 P, 2 S, 1 D$, and $2 P$, all in the $\Sigma_{g}^{+}$potential.

In order to compare to actual measured exotic masses, one must introduce spin and isospin splitting (i.e., fine structure) into the exotic multiplets by identifying significant physical effects expected to break the mass degeneracy of the multiplet, such as spin-spin couplings and spinisospin dependent operators analogous to those arising from pion exchange. The relevant operators are collected into a Hamiltonian, whose matrix elements embody the level spacing of the mass spectrum. The $1 S$ multiplet is studied this way in Ref. [24], in which it is found that the essential known phenomenological features of the lightest hidden-charm exotics $X(3872), Z_{c}(3900)$, and $Z_{c}(4020)$ emerge naturally. These techniques can also be applied directly to the $c \bar{c} s \bar{s}$ sector, as presented in a forthcoming study [25].

Here we extend this study to the $1 P$ multiplet, which as enumerated in Ref. [13] and again in Table I below, contains 28 isomultiplets. As to be discussed in Sec. III, no more than 6 of these states have been observed, and indeed, the experimental situation for even these few states remains unsettled. Nevertheless, subsequent analysis by BESIII and future measurements at Belle II and elsewhere can be expected to greatly clarify this murky picture. In fact, by minimally extending the Hamiltonian of Ref. [24] to include spin-orbit and tensor terms, we provide a large number of testable predictions for the unknown sectors of the multiplet, and with this explicit Hamiltonian in hand, one can adjust detailed fits easily to incorporate any future experimental modifications to the known spectrum. A study of the $P$-wave $\delta$ - $\bar{\delta}$ states using QCD sum rules appears in Refs. [26,27].

This paper is organized as follows. Section II summarizes the expected spectroscopy of the dynamical diquark model, leading to a detailed enumeration of all 28 isomultiplets in the $1 P$ multiplet. In Sec. III we review the current status of exotic meson candidates in the mass range of $4050-4400 \mathrm{MeV}$ (i.e., above the $1 S$ states), identifying known $P=-$ states. Special attention is given to ambiguities in the data for even the best-known $J^{P C}=1^{--} Y$ states. We then turn in Sec. IV to the development of a Hamiltonian for the $1 P$ states, starting with the form shown to be successful for describing the phenomenology of the $1 S$ states in Ref. [24]. Section V exhibits fits of the data to
TABLE I. The $28 c \bar{c} q \bar{q}^{\prime}$ isomultiplets for the $\Sigma_{g}^{+}(1 P)$ band in the dynamical diquark model. The given state notation, using the basis of Eq. (4), uniquely specifies eigenvalues of light-quark spin $s_{q \bar{q}}$, heavy-quark spin $s_{Q \bar{Q}}$, total quark spin $S$, and total angular momentum $J$. Discrete quantum numbers $P, C$ are obtained from Eq. (5). Names of $I=0$ and $I=1$ states according to Particle Data Group conventions [28] are also presented. $J_{q \bar{q}}$ [Eq. (18)] denotes eigenvalues of the total angular momentum carried by light quarks, and $\mathcal{M}_{J_{q \bar{q}}}$ [Eq. (19)] gives the amplitude of each allowed $J_{q \bar{q}}$ eigenstate within the given state.

\begin{tabular}{|c|c|c|c|c|c|c|c|c|}
\hline State & $J^{P C}$ & $I=0$ & $I=1$ & $s_{q \bar{q}}$ & $s_{Q \bar{Q}}$ & $S$ & $J_{q \bar{q}}$ & $\mathcal{M}_{J_{q \bar{q}}}$ \\
\hline$\tilde{X}_{0}^{(1)}$ & $1^{--}$ & $\psi$ & $R_{c 1}$ & 0 & 0 & 0 & 1 & +1 \\
\hline \multirow[t]{3}{*}{$\tilde{X}_{0}^{\prime(1)}$} & $1^{--}$ & $\psi$ & $R_{c 1}$ & 1 & 1 & 0 & 0 & $+\frac{1}{3}$ \\
\hline & & & & & & & 1 & $-\frac{1}{\sqrt{3}}$ \\
\hline & & & & & & & 2 & \\
\hline \multirow[t]{3}{*}{$X_{1}^{(1)}$} & $1^{--}$ & $\psi$ & $R_{c 1}$ & 1 & 1 & 1 & 0 & $-\frac{1}{\sqrt{3}}$ \\
\hline & & & & & & & 1 & \\
\hline & & & & & & & 2 & $+\frac{1}{2} \sqrt{.}$ \\
\hline \multirow[t]{3}{*}{$X_{2}^{(1)}$} & $1^{--}$ & $\psi$ & $R_{c 1}$ & 1 & 1 & 2 & 0 & $+\frac{\sqrt{5}}{3}$ \\
\hline & & & & & & & 1 & $+\frac{1}{2} \sqrt{ }$ \\
\hline & & & & & & & 2 & $+\frac{1}{6}$ \\
\hline$X_{1}^{(0)}$ & $0^{--}$ & $\psi_{0}$ & $R_{c 0}$ & 1 & 1 & 1 & 1 & +1 \\
\hline \multirow[t]{2}{*}{$X_{1}^{(2)}$} & $2^{--}$ & $\psi_{2}$ & $R_{c 2}$ & 1 & 1 & 1 & 1 & $-\frac{1}{2}$ \\
\hline & & & & & & & 2 & $+\frac{\sqrt{3}}{2}$ \\
\hline \multirow[t]{2}{*}{$X_{2}^{(2)}$} & $2^{--}$ & $\psi_{2}$ & $R_{c 2}$ & 1 & 1 & 2 & 1 & $+\frac{\sqrt{3}}{2}$ \\
\hline & & & & & & & 2 & $+\frac{1}{2}$ \\
\hline$X_{2}^{(3)}$ & $3^{--}$ & $\psi_{3}$ & $R_{c 3}$ & 1 & 1 & 2 & 2 & +1 \\
\hline$\tilde{Z}^{(0)}$ & $0^{-+}$ & $\eta_{c}$ & $\Pi_{c 0}$ & 1 & 0 & 1 & 0 & +1 \\
\hline$\tilde{Z}^{\prime(0)}$ & $0^{-+}$ & $\eta_{c}$ & $\Pi_{c 0}$ & 0 & 1 & 1 & 1 & +1 \\
\hline$\tilde{Z}^{(1)}$ & $1^{-+}$ & $\eta_{c 1}$ & $\Pi_{c 1}$ & 1 & 0 & 1 & 1 & +1 \\
\hline$\tilde{Z}^{\prime(1)}$ & $1^{-+}$ & $\eta_{c 1}$ & $\Pi_{c 1}$ & 0 & 1 & 1 & 1 & +1 \\
\hline$\tilde{Z}^{(2)}$ & $2^{-+}$ & $\eta_{c 2}$ & $\Pi_{c 2}$ & 1 & 0 & 1 & 2 & +1 \\
\hline$\tilde{Z}^{\prime(2)}$ & $2^{-+}$ & $\eta_{c 2}$ & $\Pi_{c 2}$ & 0 & 1 & 1 & 1 & +1 \\
\hline
\end{tabular}

experiment, using several hypotheses for identifying the 4 $I=0,1^{--}$states of the $1 P$ multiplet with the observed $Y$ states. We summarize briefly in Sec. VI.

\section{SPECTROSCOPY OF P-WAVE $Q \bar{Q} q \bar{q}^{\prime}$ EXOTICS}

The full spectroscopy of diquark-antidiquark $(\delta-\bar{\delta})$ exotics connected by a gluonic field of arbitrary excitation quantum numbers, and for arbitrary orbital excitations between the $\delta-\bar{\delta}$ pair, is presented in Ref. [13], and much of the content in this section mirrors Secs. III and IV from that work. As discussed in Ref. [13], the excitations of the gluonic field produce states analogous to the hybrids of ordinary quarkonium, and may be classified according to the quantum numbers provided by Born-Oppenheimer (BO) potentials. However, detailed numerical studies [23] show that such states lie above the corresponding 
BO ground-state potential (called $\Sigma_{g}^{+}$) by at least $1 \mathrm{GeV}$, comparable to the amount by which heavy-quarkonium hybrids are expected from lattice simulations to lie above ordinary quarkonium (see, e.g., Ref. [29]). Since the entire range of observed hidden-charm exotic candidates spans only about $800 \mathrm{MeV}$ [1], likely all of these states occupy the $\Sigma_{g}^{+}$BO potential, and specifically can be all accommodated by the lowest $\Sigma_{g}^{+}$levels: $1 S, 1 P, 2 S, 1 D$, and $2 P$ [23].

A detailed accounting of the possible $Q \bar{Q} q \bar{q}^{\prime}$ states (where the light quarks $q$ and $q^{\prime}$ do not necessarily carry the same flavor) is straightforward for the $S$ wave, in which the components possess no relative orbital angular momentum. In this case, any two naming conventions for the states differ only by the order in which the 4 quark spins are coupled. In the diquark basis, in which the quark spins are coupled in the order $(q Q)+(\bar{q} \bar{Q})$, the 6 possible states are denoted by [30]:

$$
\begin{gathered}
J^{P C}=0^{++}: X_{0}=\left|0_{\delta}, 0_{\bar{\delta}}\right\rangle_{0}, \quad X_{0}^{\prime}=\left|1_{\delta}, 1_{\bar{\delta}}\right\rangle_{0}, \\
J^{P C}=1^{++}: X_{1}=\frac{1}{\sqrt{2}}\left(\left|1_{\delta}, 0_{\bar{\delta}}\right\rangle_{1}+\left|0_{\delta}, 1_{\bar{\delta}}\right\rangle_{1}\right), \\
J^{P C}=1^{+-}: Z=\frac{1}{\sqrt{2}}\left(\left|1_{\delta}, 0_{\bar{\delta}}\right\rangle_{1}-\left|0_{\delta}, 1_{\bar{\delta}}\right\rangle_{1}\right), \\
Z^{\prime}=\left|1_{\delta}, 1_{\bar{\delta}}\right\rangle_{1}, \\
J^{P C}=2^{++}: X_{2}=\left|1_{\delta}, 1_{\bar{\delta}}\right\rangle_{2},
\end{gathered}
$$

where outer subscripts indicate total quark spin $S$. But the same states may be expressed in any other basis by using angular momentum recoupling coefficients; because one has a total of 4 angular momenta, the $9 j$ symbol applies. For the purposes of this work, the most useful alternate basis is that of definite heavy-quark (and light-quark) spin, $(Q \bar{Q})+(q \bar{q})$ :

$$
\begin{array}{r}
\left\langle\left(s_{q} s_{\bar{q}}\right) s_{q \bar{q}},\left(s_{Q} s_{\bar{Q}}\right) s_{Q \bar{Q}}, S \mid\left(s_{q} s_{Q}\right) s_{\delta},\left(s_{\bar{q}} s_{\bar{Q}}\right) s_{\bar{\delta}}, S\right\rangle \\
=\left(\left[s_{q \bar{q}}\right]\left[s_{Q \bar{Q}}\right]\left[s_{\delta}\right]\left[s_{\bar{\delta}}\right]\right)^{1 / 2}\left\{\begin{array}{lll}
s_{q} & s_{\bar{q}} & s_{q \bar{q}} \\
s_{Q} & s_{\bar{Q}} & s_{Q \bar{Q}} \\
s_{\delta} & s_{\bar{\delta}} & S
\end{array}\right\},
\end{array}
$$

where $[s] \equiv 2 s+1$ denotes the multiplicity of a spin-s state. Using Eqs. (1) and (2), one then obtains

$$
\begin{aligned}
J^{P C}=0^{++}: X_{0} & \equiv \frac{1}{2}\left|0_{q \bar{q}}, 0_{Q \bar{Q}}\right\rangle_{0}+\frac{\sqrt{3}}{2}\left|1_{q \bar{q}}, 1_{Q \bar{Q}}\right\rangle_{0}, \\
X_{0}^{\prime} & \equiv \frac{\sqrt{3}}{2}\left|0_{q \bar{q}}, 0_{Q \bar{Q}}\right\rangle_{0}-\frac{1}{2}\left|1_{q \bar{q}}, 1_{Q \bar{Q}}\right\rangle_{0}, \\
J^{P C}=1^{++}: X_{1} & \equiv\left|1_{q \bar{q}}, 1_{Q \bar{Q}}\right\rangle_{1}, \\
J^{P C}=1^{+-}: Z & \equiv \frac{1}{\sqrt{2}}\left(\left|1_{q \bar{q}}, 0_{Q \bar{Q}}\right\rangle_{1}-\left|0_{q \bar{q}}, 1_{Q \bar{Q}}\right\rangle_{1}\right), \\
Z^{\prime} & \equiv \frac{1}{\sqrt{2}}\left(\left|1_{q \bar{q}}, 0_{Q \bar{Q}}\right\rangle_{1}+\left|0_{q \bar{q}}, 1_{Q \bar{Q}}\right\rangle_{1}\right), \\
J^{P C}=2^{++}: X_{2} & \equiv\left|1_{q \bar{q}}, 1_{Q \bar{Q}}\right\rangle_{2} .
\end{aligned}
$$

A similar recoupling can be used to express these states in terms of equivalent heavy-light meson spins, $(q \bar{Q})+(\bar{q} Q)$.

The pairs of states $X_{0}, X_{0}^{\prime}$, and $Z, Z^{\prime}$ carry the same value of $J^{P C}$ (their discrete quantum numbers to be discussed below), and can therefore mix. One may define the equivalent unique heavy-quark spin eigenstates, which are: $X_{1}, X_{2}$, and

$$
\begin{aligned}
& \tilde{X}_{0} \equiv\left|0_{q \bar{q}}, 0_{Q \bar{Q}}\right\rangle_{0}=+\frac{1}{2} X_{0}+\frac{\sqrt{3}}{2} X_{0}^{\prime}, \\
& \tilde{X}_{0}^{\prime} \equiv\left|1_{q \bar{q}}, 1_{Q \bar{Q}}\right\rangle_{0}=+\frac{\sqrt{3}}{2} X_{0}-\frac{1}{2} X_{0}^{\prime}, \\
& \tilde{Z} \equiv\left|1_{q \bar{q}}, 0_{Q \bar{Q}}\right\rangle_{1}=\frac{1}{\sqrt{2}}\left(Z^{\prime}+Z\right), \\
& \tilde{Z}^{\prime} \equiv\left|0_{q \bar{q}}, 1_{Q \bar{Q}}\right\rangle_{1}=\frac{1}{\sqrt{2}}\left(Z^{\prime}-Z\right) .
\end{aligned}
$$

Up to this point, the light quarks $q$ and $\bar{q}$ have been taken to carry the same flavor. Allowing for a potentially distinct light flavor $\bar{q}^{\prime}$ for the antiquark then introduces the isospin quantum number into the analysis. ${ }^{1}$ Each state in Eqs. (1) or (4) then occurs in $I=0$ and $I=1$ variants, doubling the list to 12 possible isomultiplets. Experimentally, both $I=0$ and $I=1$ exotic candidates have been observed, making the inclusion of isospin an essential ingredient for an accurate phenomenological analysis. In the nomenclature adopted by the Particle Data Group (PDG) [28], the observed $S$-wave states $X_{0} / X_{0}^{\prime}, X_{1}, Z / Z^{\prime}$, and $X_{2}$ with $I=0$ are to be denoted as $\chi_{c 0}, \chi_{c 1}, h_{c}$, and $\chi_{c 2}$, respectively, while those with $I=1$ are to be denoted as $W_{c 0}, W_{c 1}, Z_{c}$, and $W_{c 2}$, respectively.

Allowing now for an orbital excitation quantum number $L$ between the $\delta-\bar{\delta}$ pair (but not within either of $\delta$ or $\bar{\delta}$ ), the parity eigenvalue $P$ is simply the usual spatial factor $(-1)^{L}$, since the intrinsic parity factor -1 attached to an antiquark appears twice. One immediately notes that all 12 states in the $1 S$ band have $P=+$; and in the hidden-charm sector, this feature is in accord with experiment. Namely, all exotic candidates supported by strong experimental evidence and with known parity, stretching from the $X(3872)$ up to a mass of $4200 \mathrm{MeV}$, have $P=+$ and fit nicely into the $1 S$ level. In fact, the explicit numerical calculations of Ref. [23] show that the centroids of the $1 S$ and $1 P$ multiplets are separated by about $370 \mathrm{MeV}$. Meanwhile, Ref. [24] presents an analysis of mass splittings among the 12 hidden-charm $1 S$ states (both known and predicted), and shows that the $X(3872)$ can emerge naturally as the lightest member of the multiplet. Building upon this analysis, the current paper focuses upon the $1 P$ states, all of which carry $P=-$.

\footnotetext{
${ }^{1}$ If the $s$ quark is also considered light, one may generalize to consider states in $\mathrm{SU}(3)_{\text {flavor }}$ multiplets.
} 
The charge-conjugation quantum number $C$ is most easily determined in the $(Q \bar{Q})+(q \bar{q})$ basis, since the exchange of each quark with its antiquark requires both a spatial inversion factor $(-1)^{L}$ (exchanging $\delta \leftrightarrow \bar{\delta}$ ) and spin-exchange factors $(-1)^{s_{Q \bar{Q}}+1}$ and $(-1)^{s_{q \bar{q}}+1}$. Thus

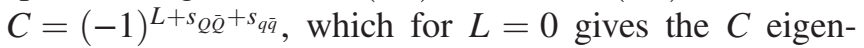
values listed in Eq. (4). The same analysis holds if the state may be considered in a molecular picture, for which the orbital excitation $L$ connects the heavy-light components $(q \bar{Q})+(\bar{q} Q)$.

Lastly, $C$ is a good quantum number only for states with $q^{\prime}=q$, i.e., the $I_{3}=0$ (neutral) members of isomultiplets. More generally, the good quantum number relevant for both neutral and charged states is $G$ parity, $G=(-1)^{I} C$, where $C$ refers to the neutral member of the isomultiplet. In summary, one has

$$
\begin{aligned}
P & =(-1)^{L}, \\
C & =(-1)^{L+s_{Q \bar{Q}}+s_{q \bar{q}}} \quad \text { (neutral states), } \\
G & =(-1)^{I+L+s_{Q \bar{Q}}+s_{q \bar{q}}} .
\end{aligned}
$$

Considering, finally, the $P$-wave states that form the subject of this paper, one enumerates them ${ }^{2}$ simply by combining the $S$-wave "core" states of Eqs. (1) or (4) (generically denoted here by $Y$ ) with a unit of orbital angular momentum, $L=1$, and imposes the usual rules of angular momentum addition to form eigenstates of total $J$. The notation introduced in Ref. [13] then reads $Y_{L}^{(J)}$; since we are interested only in $P$-wave states, the subscript $L=1$ is suppressed. Using the heavy-quark basis of Eq. (4), one obtains the 28 isomultiplets listed in Table I, 14 with $I=0$ and 14 with $I=1$.

\section{EXPERIMENTAL REVIEW OF RELEVANT STATES}

The hidden-charm exotic-meson candidates with masses above the well-known $X(3872), Z_{c}(3900)$, and $Z_{c}(4020)$, but below that of the $Z_{c}(4430)$, are listed in Table II. This range has been chosen in order to eliminate many states clearly established to carry positive parity (such as those just listed), but also to bracket the well-known $J^{P C}=1^{--}$ states $Y(4230), Y(4260), Y(4390)$, and $Y(4360)$. The higher-mass $1^{--} Y(4626)[32,33]$ and $Y(4660)$ are also included, being the only hidden-charm exotic candidates with clearly established negative parity that lie outside this range.

The only other observed $P=-$ exotic candidate, the (unconfirmed) $Z_{c}(4240)$, carries quark-model exotic quantum numbers $J^{P C}=0^{--}$as well as $I=1$, and also lies in

\footnotetext{
${ }^{2}$ The number of $P$-wave $\delta$ - $\bar{\delta}$ states was first counted in Ref. [31], and the corresponding counting for all partial waves appears in Ref. [13].
}

the range $4020-4480 \mathrm{MeV}$. The only states inside this range confirmed to carry $P=+$ are $\chi_{c 1}(4140)$ and $\chi_{c 1}(4274)$ (both $1^{++}$), and $Z_{c}(4200)\left(1^{+-}\right)$. However, the former 2 states have only been seen in decays to $J / \psi \phi$, and may therefore be $c \bar{c} s \bar{s}$ states [34] (and similarly for the $P=$ $-Y(4626)$ due to its $D_{s}^{+} D_{s 1, s 2}^{-}$decays $\left.[32,33]\right)$, while the existence of $Z_{c}(4200)$ remains unconfirmed.

The currently known data set of $P=-$ exotics therefore consists of the 6 states $Y(4230), Z_{c}(4240), Y(4260)$, $Y(4360), Y(4390), Y(4626)$, and $Y(4660) . Z_{c}(4240)$ has only been observed in the LHCb experiment that confirmed the existence of the $1^{+-} Z_{c}(4430)$ [35], and both states are clearly $I=1$ because they are seen in decays to $\psi(2 S) \pi^{ \pm}$. Yet even the well-studied $Y$ states present a number of mysteries. Each one has only been produced either via initial-state radiation processes $\left(e^{+} e^{-} \rightarrow \gamma Y\right)$ at $B A B A R$, CLEO, and Belle, or directly via $e^{+} e^{-} \rightarrow Y$ at BESIII. Unlike the conventional charmonium $1^{--}$states, none of them produce obvious peaks in $R\left(e^{+} e^{-} \rightarrow\right.$ hadrons) (see Fig. 51.3 in Ref. [28]). Their only open-charm decays yet observed are $Y(4230) \rightarrow \pi^{+} D^{0} D^{*-}$ [36], $Y(4626) \rightarrow$ $D_{s}^{+} D_{s 1, s 2}^{-}$[32,33], and $Y(4660) \rightarrow \Lambda_{c}^{+} \Lambda_{c}^{-}$, and this latter mode has not been confirmed to belong specifically to $Y(4660)$. $Y(4230), Y(4260), Y(4360)$, and $Y(4626)$ are confirmed to be $I=0$ due to their observed decays to $\chi_{c 0} \omega$, $J / \psi f_{0}(980), \psi(2 S) \pi^{0} \pi^{0}$, and $D_{s} D_{s 1, s 2}$, respectively. But $Y(4390)$ and $Y(4660)$ (not counting the latter state's possible $\Lambda_{c}^{+} \Lambda_{c}^{-}$mode) do not yet have confirmed isospin quantum numbers, and could easily carry $I=1$, due to their production via a virtual photon.

The pattern of observed $Y$ decays to conventional charmonium is itself quite confusing: Referring to Table II, the $Y(4230)$ has been seen to decay to $\psi(2 S)$, $\chi_{c 0}$, and $h_{c}$, while $Y(4260)$ is so far only observed in channels decaying (eventually) to $J / \psi ; Y(4360)$ to both $J / \psi$ and $\psi(2 S) ; Y(4660)$ only to $\psi(2 S)$; and $Y(4390)$ only to $h_{c}$. Recent data from BESIII [37] show that $Y(4230)$ and $Y(4390)$ both decay to $J / \psi \eta$, which restricts $Y(4390)$ to $I=0$.

However, closer inspection of the data introduces yet another level of ambiguity. BESIII, having the world's most complete data set on $1^{--}$states, does not confirm the location of $Y(4260)$ given by earlier experiments (which give well-clustered average values $m=4251 \pm 6 \mathrm{MeV}$, $\Gamma=120 \pm 12 \mathrm{MeV}$ ), and instead reports $m=4222.0 \pm$ $3.4 \mathrm{MeV}$ and $\Gamma=44.1 \pm 4.7 \mathrm{MeV}$ [38]. Note that the former numbers appear in $e^{+} e^{-} \rightarrow \gamma Y$ measurements and the latter directly from $e^{+} e^{-} \rightarrow Y$. Indeed, the BESIII numbers for $Y(4260)$ actually agree rather well with those given in Table II for $Y(4230)$, which is especially true now that the current values extracted from $\chi_{c 0} \omega$ have been updated [39]: $m=4218.5 \pm 4.3 \mathrm{MeV}$ and $\Gamma=28.2 \pm 4.2 \mathrm{MeV}$. Similarly, the BESIII mass measurements ascribed to $Y(4360)$ give two widely separated 
TABLE II. All charmoniumlike exotic-meson candidates catalogued by the Particle Data Group (PDG) [28] to lie in mass above $Z_{c}(4020)$ and below $Z_{c}(4430)$. Also included are $Y(4626)$ and $Y(4660)$, the only other charmoniumlike exotic mesons observed to carry $P=-$. Both the particle name most commonly used in the literature and its label as given in the PDG are listed.

\begin{tabular}{|c|c|c|c|c|c|}
\hline Particle & PDG label & $I^{G} J^{P C}$ & Mass $[\mathrm{MeV}]$ & Width $[\mathrm{MeV}]$ & Production and decay \\
\hline$Z_{c}(4050)$ & $X(4050)^{ \pm}$ & $1^{-} ?^{?+}$ & $4051_{-40}^{+24}$ & $82_{-28}^{+50}$ & $B \rightarrow K Z ; Z \rightarrow \pi^{ \pm} \chi_{c 1}$ \\
\hline$Z_{c}(4055)$ & $X(4055)^{ \pm}$ & $1^{+} ?^{?-}$ & $4054 \pm 3.2$ & $45 \pm 13$ & $e^{+} e^{-} \rightarrow \gamma \pi^{\mp} Z ; Z \rightarrow \pi^{ \pm} \psi(2 S)$ \\
\hline$Z_{c}(4100)$ & $X(4100)^{ \pm}$ & $1^{-} ?^{? ?}$ & $4096 \pm 28$ & $152_{-70}^{+80}$ & $B \rightarrow K Z ; Z \rightarrow \pi^{ \pm} \eta_{c}$ \\
\hline$Y(4140)$ & $\chi_{c 1}(4140)$ & $0^{+} 1^{++}$ & $4146.8 \pm 2.4$ & $22_{-7}^{+8}$ & $\left.\begin{array}{r}B \rightarrow K Y \\
p \bar{p} \rightarrow Y+\text { anything }\end{array}\right\} ; Y \rightarrow \phi J / \psi$ \\
\hline$X(4160)$ & $X(4160)$ & $? ? ? ?$ & $4156_{-25}^{+29}$ & $139_{-60}^{+110}$ & $e^{+} e^{-} \rightarrow J / \psi+X ; X \rightarrow D^{*} \bar{D}^{*}$ \\
\hline$Z_{c}(4200)$ & $Z_{c}(4200)$ & $1^{+} 1^{+-}$ & $4196_{-32}^{+35}$ & $370_{-150}^{+100}$ & $B \rightarrow K Z ; Z \rightarrow \pi^{ \pm} J / \psi$ \\
\hline$Y(4230)$ & $\psi(4230)$ & $0^{-} 1^{--}$ & $4218_{-4}^{+5}$ & $59_{-10}^{+12}$ & $e^{+} e^{-} \rightarrow Y ; Y \rightarrow\left\{\begin{array}{l}\omega \chi_{c 0} \\
\eta J / \psi \\
\pi^{+} \pi^{-} h_{c} \\
\pi^{+} \pi^{-} \psi(2 S) \\
\pi^{+} D^{0} D^{*-}\end{array}\right.$ \\
\hline$Z_{c}(4240)$ & $R_{c 0}(4240)$ & $1^{+} 0^{--}$ & $4239_{-21}^{+50}$ & $220_{-90}^{+120}$ & $B \rightarrow K Z ; Z \rightarrow \pi^{ \pm} \psi(2 S)$ \\
\hline$Z_{c}(4250)$ & $X(4250)^{ \pm}$ & $1^{-} ?^{?+}$ & $4248_{-50}^{+190}$ & $177_{-70}^{+320}$ & $B \rightarrow K Z ; Z \rightarrow \pi^{ \pm} \chi_{c 1}$ \\
\hline$Y(4260)$ & $\psi(4260)$ & $0^{-} 1^{--}$ & $4230 \pm 8$ & $55 \pm 19$ & $e^{+} e^{-} \rightarrow \gamma Y$ or $Y ; Y \rightarrow\left\{\begin{array}{l}\pi^{+} \pi^{-} J / \psi \\
f_{0}(980) J / \psi \\
\pi^{\mp} Z_{c}^{ \pm}(3900) \\
K^{+} K^{-} J / \psi\end{array}\right.$ \\
\hline$Y(4274)$ & $\chi_{c 1}(4274)$ & $0^{+} 1^{++}$ & $4274_{-6}^{+8}$ & $49 \pm 12$ & $B \rightarrow K Y ; Y \rightarrow \phi J / \psi$ \\
\hline$X(4350)$ & $X(4350)$ & $0^{+} ? ?+$ & $4351 \pm 5$ & $13_{-10}^{+18}$ & $\gamma \gamma \rightarrow X ; X \rightarrow \phi J / \psi$ \\
\hline$Y(4360)$ & $\psi(4360)$ & $0^{-} 1^{--}$ & $4368 \pm 13$ & $96 \pm 7$ & $e^{+} e^{-} \rightarrow \gamma Y$ or $Y ; Y \rightarrow\left\{\begin{array}{l}\pi^{+} \pi^{-} \psi(2 S) \\
\pi^{0} \pi^{0} \psi(2 S)\end{array}\right.$ \\
\hline$Y(4390)$ & $\psi(4390)$ & $0^{-} 1^{--}$ & $4392 \pm 7$ & $140_{-21}^{+16}$ & $e^{+} e^{-} \rightarrow Y ; Y \rightarrow\left\{\begin{array}{l}\eta J / \psi \\
\pi^{+} \pi^{-} h_{c}\end{array}\right.$ \\
\hline$Y(4626)$ & $\psi(4626)$ & $0^{-} 1^{--}$ & $4626 \pm 6$ & $49_{-12}^{+14}$ & $\begin{aligned} e^{+} e^{-} \rightarrow & \gamma Y ; Y \rightarrow D_{s}^{+} D_{s 1}(2536)^{-} \\
& {\left[\text {or } D_{s}^{+} D_{s 2}^{*}(2573)^{-}\right] }\end{aligned}$ \\
\hline$Y(4660)$ & $\psi(4660)$ & $0^{-} 1^{--}$ & $4643 \pm 9$ & $72 \pm 11$ & $e^{+} e^{-} \rightarrow\left\{\begin{array}{l}\gamma Y ; Y \rightarrow \pi^{+} \pi^{-} \psi(2 S) \\
Y ; Y \rightarrow \Lambda_{c}^{+} \Lambda_{c}^{-}\end{array}\right.$ \\
\hline
\end{tabular}

values, $4320 \pm 13 \mathrm{MeV}$ for $e^{+} e^{-} \rightarrow J / \psi \pi^{+} \pi^{-}$[38] versus $4386 \pm 4 \mathrm{MeV}$ for $e^{+} e^{-} \rightarrow \psi(2 S) \pi^{+} \pi^{-}[40]$.

It is worth noting, in such a fluid experimental landscape, that signals for decays into particular channels currently ascribed to a single resonance might actually represent observations of more than one resonance closely spaced in mass. For example, the isolated low-mass measurement of $e^{+} e^{-} \rightarrow J / \psi \pi^{+} \pi^{-}$from Ref. [38] could easily signal a resonance distinct from $Y(4360)$, and indeed, $I=1$ cannot yet be ruled out for this mode. Until such time as this mass range is fully explored in the charged sector, ${ }^{3}$ a definitive description of the splitting of quartets of states into $I=0$ and $I=1$ multiplets remains unavailable.

Lastly, we do not consider in this work the broad $1^{--} J / \psi \pi^{+} \pi^{-}$resonance $Y(4008)$ claimed by Belle [42], as it has not been seen by other experiments, particularly in

\footnotetext{
${ }^{3}$ For example, a very recent experiment by $\mathrm{LHCb}[41]$ shows resonant structure in $J / \psi \pi^{-}$around 4200 and around $4600 \mathrm{MeV}$.
}

the precise scan of BESIII [38]. We also do not consider the charged [40] and neutral [43] charmoniumlike $\psi(2 S) \pi$ "structures" observed by BESIII around $4035 \mathrm{MeV}$, which may be the same as $Z_{c}(4055)$ [28].

\section{MASS HAMILTONIAN OPERATORS}

\section{A. Operators appearing for $1 S$ and $1 P$}

The analysis of the $1 S$ states in Ref. [24] uses a Hamiltonian consisting of only 3 operators,

$H=M_{0}+2 \kappa_{q Q}\left(\mathbf{s}_{q} \cdot \mathbf{s}_{Q}+\mathbf{s}_{\bar{q}} \cdot \mathbf{s}_{\bar{Q}}\right)+V_{0} \boldsymbol{\tau}_{q} \cdot \boldsymbol{\tau}_{\bar{q}} \boldsymbol{\sigma}_{q} \cdot \boldsymbol{\sigma}_{\bar{q}}$,

where $M_{0}$ is the common multiplet mass; the $\kappa_{q Q}$ term indicates that the only isospin-blind spin couplings are taken to be only those between pairs of quarks within the same diquark: $q \leftrightarrow Q$ in $\delta$ and $\bar{q} \leftrightarrow \bar{Q}$ in $\bar{\delta}$; and $V_{0}$ is a spin-isospin operator identical in form to that appearing in the canonical one-pion exchange between nucleons, except that it is taken to act only between the light quarks $q, \bar{q}$ 
rather than between the full diquarks. Isospin-blind spinspin couplings between quarks within different diquarks (e.g., $q$ and $\bar{Q}$ ) are ignored in Ref. [24] under the assumption that $\delta, \bar{\delta}$ are somewhat separated quasiparticles within the hadron, and thus isospin-blind interactions between $\delta$ and $\bar{\delta}$ are assumed to be relatively small compared to the spin couplings inside of them. An alternative choice for the dominant isospin-dependent operator is also considered in Ref. [24],

$$
\Delta H=V_{1} \boldsymbol{\tau}_{q} \cdot \boldsymbol{\tau}_{\bar{q}} \boldsymbol{\sigma}_{\delta} \cdot \boldsymbol{\sigma}_{\bar{\delta}},
$$

in which the isospin-dependent interaction is chosen to couple to the full $\delta$ or $\bar{\delta}$ as a unit (noting that $Q, \bar{Q}$ are isosinglets, so that $\boldsymbol{\tau}_{\delta}=\boldsymbol{\tau}_{q}$ ). However, such an operator leaves the $J^{P C}=1^{++}$states with $I=0$ and $I=1$ in the $1 S$ multiplet degenerate in mass. Since one of the key experimental properties of the $1^{++} X(3872)$ is its lack of charged partners [44], the operator in Eq. (7) cannot give the dominant isospin-dependent effect for the $1 S$ states. We inferred in Ref. [24] that the isospin-dependent couplings, at least in the $S$ wave, appear to see only the light quarks, and do not view the diquarks as pointlike constituents. Indeed, one of the major thrusts of Ref. [24] is a study showing that the spectrum of the diquark model is quite robust when the $\delta, \bar{\delta}$ are endowed with wave functions of significant spatial extent compared to the size of the full hadron and begin to overlap. In such a situation, it is quite natural that isospin-dependent couplings are sensitive only to the light quarks $q \bar{q}^{\prime}$, independent of the heavy quarks $Q \bar{Q}$.

The Hamiltonian of Eq. (6) is shown in [24] to accommodate the known $P=+$ exotics $X(3872), Z_{c}(3900)$, and $Z_{c}(4020)$, while predicting the masses of the other 9 yetunseen states in the $1 S$ multiplet in such a way that the $X(3872)$ emerges naturally as the lightest state, $Z_{c}(3900)$ preferentially decays to $J / \psi \pi$, and $Z_{c}(4020)$ preferentially decays to $h_{c} \pi$, in complete accord with experiment. Nevertheless, the Hamiltonian of Eq. (6) contains only operators expected to provide the most significant physical effects. While it is possible to fit the 3 constants $M_{0}, \kappa_{q Q}$, $V_{0}$ using nothing but the 3 PDG-averaged mass eigenvalues of $X(3872), Z_{c}(3900)$, and $Z_{c}(4020)$, and indeed such a restrictive fit gives results virtually identical to those in Ref. [24]:

$$
\begin{aligned}
M_{0}(1 S) & =3988.69 \mathrm{MeV}, \\
\kappa_{q Q}(1 S) & =17.89 \mathrm{MeV}, \\
V_{0}(1 S) & =33.04 \mathrm{MeV},
\end{aligned}
$$

this fit ignores the effect of subleading operators that can have a significant effect upon small mass splittings and mixing angles, as well as the inevitable shifting of PDG central mass values expected to occur as newer measurements of these masses from subsequent experiments are published. For example, $\kappa_{q Q}$ is particularly sensitive to the $Z_{c}(3900)$ mass, and would more than double if its mass measurement increased by only $10 \mathrm{MeVIn}$ addition, the values in Eq. (8) apply only to states in the $1 S$ multiplet and must be re-evaluated for the $1 P$ state, since they represent expectation values over wave functions that vary for states of different quantum numbers.

\section{B. Operators first appearing for $\mathbf{1 P}$}

Turning now to the $1 P$ states, the multiplet-average mass $M_{0}(1 P)$ can be obtained either by using mass eigenvalues of $P=-$ states alone, or by applying the simulations of Ref. [23] and fixing as an initial point the value of the multiplet-average mass $M_{0}(1 S)$ from Eq. (8). Using the latter approach, and depending upon which specific lattice simulation is used to obtain the $\Sigma_{g}^{+} \mathrm{BO}$ potential, one obtains

$$
M_{0}(1 P)=4358-4361 \mathrm{MeV} .
$$

In a purely phenomenological analysis [30] spanning the two multiplets, the $1 P-1 S$ splitting is provided by the operator $\mathbf{L}^{2}$. The next most significant $L$-dependent contribution is expected to arise from the spin-orbit term,

$$
\Delta H_{L S}=V_{L S} \mathbf{L} \cdot \mathbf{S}
$$

noting that the analogous parameter $V_{L S}$ defined in Ref. [30] is a factor of $-\frac{1}{2}$ as large as the one defined in Eq. (10). Explicitly, its matrix elements contribute to the mass an amount

$$
\Delta M_{L S}=\frac{V_{L S}}{2}[J(J+1)-L(L+1)-S(S+1)] .
$$

The parameter $\kappa_{q Q}$ from Eq. (6), providing matrix elements contributing to the mass as

$$
\Delta M_{\kappa_{q Q}}=\kappa_{q Q}\left[s_{\delta}\left(s_{\delta}+1\right)+s_{\bar{\delta}}\left(s_{\bar{\delta}}+1\right)-3\right],
$$

and representing spin-only couplings within a diquark, might be expected to vary with the mass of the heavy quark $Q,{ }^{4}$ but inasmuch as the diquarks $\delta, \bar{\delta}$ are compact and well separated within the hadron-which is not such a clear-cut proposition, in light of the discussion following Eq. (7) one would naively expect $\kappa_{q Q}$ to be roughly the same for all multiplets of a fixed flavor content. However, as seen below, the best fits to $\kappa_{q Q}$ in the $1 P$ sector favor rather larger values. Nevertheless, in light of both the sensitivity of $\kappa_{q Q}(1 S)$ to $m_{Z_{c}(3900)}$ noted above, and of questions about

\footnotetext{
${ }^{4}$ In this work $Q=c$, but the same set of operators (with different numerical values for the coefficients) is expected to apply to hidden-bottom and $B_{c}$-like exotic systems.
} 
the detailed consequences of finite diquark size, such larger values need not be construed as problematic.

The parameter $V_{0}$ from Eq. (6), providing contributions to the mass of the form

$$
\Delta M_{V_{0}}=V_{0}[2 I(I+1)-3]\left[2 s_{q \bar{q}}\left(s_{q \bar{q}}+1\right)-3\right],
$$

is introduced in Ref. [24] to represent a pionlike coupling in the colored environment of the flux tube connecting the $\delta-\bar{\delta}$ pair. As is well known, the corresponding operator representing pion exchange between two nucleons has a positive coefficient, indicating attractive $I=1$, spin-singlet and $I=0$, spin-triplet channels. The positive fit value of $V_{0}(1 S)$ in Eq. (8) suggests a similar pattern of isospin coupling for the $S$-wave $\delta$ - $\bar{\delta}$ states. In considering $P$-wave and higher states, we also introduce the isospin-dependent tensor operator,

$$
\Delta H_{T}=V_{T} \boldsymbol{\tau}_{q} \cdot \boldsymbol{\tau}_{\bar{q}} S_{12}^{(q \bar{q})},
$$

where the tensor operator $S_{12}$ is defined by

$$
S_{12} \equiv 3 \sigma_{1} \cdot \boldsymbol{r} \sigma_{2} \cdot \boldsymbol{r} / r^{2}-\sigma_{1} \cdot \sigma_{2}
$$

For the purpose of this work, we consider only the tensor operator $S_{12}^{(q \bar{q})}$, in which the spin operators in Eq. (15) refer only to the light quarks, not the full diquarks. We argue, just as after Eq. (7), that the dominant isospin-dependent tensor operator should be sensitive only to light-quark spins; nevertheless, for completeness we tabulate in the Appendix the $P$-wave multiplet matrix elements for the alternative tensor operator

$$
\Delta H_{T}^{\prime}=V_{T}^{\prime} \boldsymbol{\tau}_{q} \cdot \boldsymbol{\tau}_{\bar{q}} S_{12}^{(\delta \bar{\delta})}
$$

The matrix elements of $S_{12}$ are well known in the literature (e.g., Ref. [45]). Defining for the generic case $\mathbf{J} \equiv \mathbf{L}+\mathbf{S}$, the distinct values are

$$
\begin{gathered}
S_{12}\left|L=J, S=0, J, J_{z}\right\rangle=0, \\
\left\langle L^{\prime}=J, S=1, J, J_{z}\left|S_{12}\right| L=J, S=1, J, J_{z}\right\rangle=2, \\
\left\langle L^{\prime}=J-1, S=1, J, J_{z}\left|S_{12}\right| L=J-1, S=1, J, J_{z}\right\rangle=-\frac{2(J-1)}{2 J+1}, \\
\left\langle L^{\prime}=J+1, S=1, J, J_{z}\left|S_{12}\right| L=J+1, S=1, J, J_{z}\right\rangle=-\frac{2(J+2)}{2 J+1}, \\
\left\langle L^{\prime}=J+1, S=1, J, J_{z}\left|S_{12}\right| L=J-1, S=1, J, J_{z}\right\rangle=\frac{6 \sqrt{J(J+1)}}{2 J+1} .
\end{gathered}
$$

For the tensor operator $S_{12}^{(q \bar{q})}$ of Eq. (14), neither the basis in terms of $s_{q \bar{q}}, s_{Q \bar{Q}}$ spins nor $s_{\delta}, s_{\bar{\delta}}$ spins is convenient for the computation of matrix elements. Instead, the recoupling of the orbital angular momentum directly to the light-quark spin,

$$
\mathbf{J}_{q \bar{q}} \equiv \mathbf{L}+\mathbf{s}_{q \bar{q}}
$$

is necessary. ${ }^{5}$ The amplitudes for this recoupling are given by $6 j$ symbols:

$$
\begin{aligned}
\mathcal{M}_{J_{q \bar{q}}} & \equiv\left\langle\left(L, s_{q \bar{q}}\right), J_{q \bar{q}}, s_{Q \bar{Q}}, J \mid L,\left(s_{q \bar{q}}, s_{Q \bar{Q}}\right), S, J\right\rangle \\
& =(-1)^{L+s_{q \bar{q}}+s_{Q \bar{Q}}+J} \sqrt{\left[J_{q \bar{q}}\right][S]}\left\{\begin{array}{lll}
L & s_{q \bar{q}} & J_{q \bar{q}} \\
s_{Q \bar{Q}} & J & S
\end{array}\right\},
\end{aligned}
$$

\footnotetext{
${ }^{5}$ This procedure implicitly assumes no orbital angular momentum within either $\delta$ or $\bar{\delta}$, so that $L_{q \bar{q}}=L$.
}

and are tabulated for the states of the $P$-wave multiplet in Table I.

In summary, the full Hamiltonian adopted for the 28 isomultiplets of the $1 P$ multiplet is the sum of the 5 operators in Eqs. (6), (10), and (14):

$$
\begin{aligned}
H= & M_{0}+2 \kappa_{q Q}\left(\mathbf{s}_{q} \cdot \mathbf{s}_{Q}+\mathbf{s}_{\bar{q}} \cdot \mathbf{s}_{\bar{Q}}\right)+V_{L S} \mathbf{L} \cdot \mathbf{S} \\
& +V_{0} \boldsymbol{\tau}_{q} \cdot \boldsymbol{\tau}_{\bar{q}} \boldsymbol{\sigma}_{q} \cdot \boldsymbol{\sigma}_{\bar{q}}+V_{T} \boldsymbol{\tau}_{q} \cdot \boldsymbol{\tau}_{\bar{q}} S_{12}^{(q \bar{q})} .
\end{aligned}
$$

We now present the mass expressions for the $1 P$ states, listed in the same order as in Table I, for both $I=0$ and $I=1$. Matrices indicate sets of states degenerate in $J^{P C}$; for example, the mixing matrix element between the $I=0$, $1^{--}$states $\tilde{X}_{0}^{(1)}$ and $X_{1}^{(1)}$ is $-9 V_{T} \sqrt{3 / 5}$. Equations (21)(34), written in the basis of good total heavy-quark and light-quark spin eigenvalues, represent the central theoretical results of this work; in the next section we confront them with existing data. 
$M_{1^{--}}^{I=0}=M_{0}\left(\begin{array}{cccc}1 & 0 & 0 & 0 \\ 0 & 1 & 0 & 0 \\ 0 & 0 & 1 & 0 \\ 0 & 0 & 0 & 1\end{array}\right)+\kappa_{q Q}\left(\begin{array}{cccc}0 & -\sqrt{3} & 0 & 0 \\ -\sqrt{3} & -2 & 0 & 0 \\ 0 & 0 & -1 & 0 \\ 0 & 0 & 0 & 1\end{array}\right)-V_{L S}\left(\begin{array}{cccc}0 & 0 & 0 & 0 \\ 0 & 0 & 0 & 0 \\ 0 & 0 & 1 & 0 \\ 0 & 0 & 0 & 3\end{array}\right)-3 V_{0}\left(\begin{array}{cccc}-3 & 0 & 0 & 0 \\ 0 & 1 & 0 & 0 \\ 0 & 0 & 1 & 0 \\ 0 & 0 & 0 & 1\end{array}\right)$

$$
-3 V_{T}\left(\begin{array}{cccc}
0 & 0 & 0 & 0 \\
0 & 0 & 0 & -\frac{4}{\sqrt{5}} \\
0 & 0 & -1 & 3 \sqrt{\frac{3}{5}} \\
0 & -\frac{4}{\sqrt{5}} & 3 \sqrt{\frac{3}{5}} & -\frac{7}{5}
\end{array}\right),
$$

$M_{1}^{I=--}=M_{0}\left(\begin{array}{cccc}1 & 0 & 0 & 0 \\ 0 & 1 & 0 & 0 \\ 0 & 0 & 1 & 0 \\ 0 & 0 & 0 & 1\end{array}\right)+\kappa_{q Q}\left(\begin{array}{cccc}0 & -\sqrt{3} & 0 & 0 \\ -\sqrt{3} & -2 & 0 & 0 \\ 0 & 0 & -1 & 0 \\ 0 & 0 & 0 & 1\end{array}\right)-V_{L S}\left(\begin{array}{llll}0 & 0 & 0 & 0 \\ 0 & 0 & 0 & 0 \\ 0 & 0 & 1 & 0 \\ 0 & 0 & 0 & 3\end{array}\right)+V_{0}\left(\begin{array}{cccc}-3 & 0 & 0 & 0 \\ 0 & 1 & 0 & 0 \\ 0 & 0 & 1 & 0 \\ 0 & 0 & 0 & 1\end{array}\right)$

$$
+V_{T}\left(\begin{array}{cccc}
0 & 0 & 0 & 0 \\
0 & 0 & 0 & -\frac{4}{\sqrt{5}} \\
0 & 0 & -1 & 3 \sqrt{\frac{3}{5}} \\
0 & -\frac{4}{\sqrt{5}} & 3 \sqrt{\frac{3}{5}} & -\frac{7}{5}
\end{array}\right),
$$

$$
\begin{aligned}
& M_{0^{--}}^{I=0}=M_{0}-\kappa_{q Q}-2 V_{L S}-3 V_{0}-6 V_{T}, \\
& M_{0^{--}}^{I=1}=M_{0}-\kappa_{q Q}-2 V_{L S}+V_{0}+2 V_{T} \\
& M_{2^{--}}^{I=0}=M_{0}\left(\begin{array}{ll}
1 & 0 \\
0 & 1
\end{array}\right)+\kappa_{q Q}\left(\begin{array}{cc}
-1 & 0 \\
0 & 1
\end{array}\right)+V_{L S}\left(\begin{array}{cc}
1 & 0 \\
0 & -1
\end{array}\right)-3 V_{0}\left(\begin{array}{ll}
1 & 0 \\
0 & 1
\end{array}\right)-\frac{3}{5} V_{T}\left(\begin{array}{cc}
1 & -3 \sqrt{3} \\
-3 \sqrt{3} & 7
\end{array}\right), \\
& M_{2^{--}}^{I=1}=M_{0}\left(\begin{array}{ll}
1 & 0 \\
0 & 1
\end{array}\right)+\kappa_{q Q}\left(\begin{array}{cc}
-1 & 0 \\
0 & 1
\end{array}\right)+V_{L S}\left(\begin{array}{cc}
1 & 0 \\
0 & -1
\end{array}\right)+V_{0}\left(\begin{array}{ll}
1 & 0 \\
0 & 1
\end{array}\right)+\frac{1}{5} V_{T}\left(\begin{array}{cc}
1 & -3 \sqrt{3} \\
-3 \sqrt{3} & 7
\end{array}\right) \\
& M_{3^{--}}^{I=0}=M_{0}+\kappa_{q Q}+2 V_{L S}-3 V_{0}+\frac{6}{5} V_{T}, \\
& M_{3^{--}}^{I=1}=M_{0}+\kappa_{q Q}+2 V_{L S}+V_{0}-\frac{2}{5} V_{T}, \\
& M_{0^{-+}}^{I=0}=M_{0}\left(\begin{array}{ll}
1 & 0 \\
0 & 1
\end{array}\right)+\kappa_{q Q}\left(\begin{array}{ll}
0 & 1 \\
1 & 0
\end{array}\right)-2 V_{L S}\left(\begin{array}{ll}
1 & 0 \\
0 & 1
\end{array}\right)-3 V_{0}\left(\begin{array}{cc}
-3 & 0 \\
0 & 1
\end{array}\right)+12 V_{T}\left(\begin{array}{ll}
1 & 0 \\
0 & 0
\end{array}\right), \\
& M_{0^{-+}}^{I=1}=M_{0}\left(\begin{array}{ll}
1 & 0 \\
0 & 1
\end{array}\right)+\kappa_{q Q}\left(\begin{array}{ll}
0 & 1 \\
1 & 0
\end{array}\right)-2 V_{L S}\left(\begin{array}{ll}
1 & 0 \\
0 & 1
\end{array}\right)+V_{0}\left(\begin{array}{cc}
-3 & 0 \\
0 & 1
\end{array}\right)-4 V_{T}\left(\begin{array}{ll}
1 & 0 \\
0 & 0
\end{array}\right), \\
& M_{1^{-+}}^{I=0}=M_{0}\left(\begin{array}{ll}
1 & 0 \\
0 & 1
\end{array}\right)+\kappa_{q Q}\left(\begin{array}{ll}
0 & 1 \\
1 & 0
\end{array}\right)-V_{L S}\left(\begin{array}{ll}
1 & 0 \\
0 & 1
\end{array}\right)-3 V_{0}\left(\begin{array}{cc}
-3 & 0 \\
0 & 1
\end{array}\right)-6 V_{T}\left(\begin{array}{ll}
1 & 0 \\
0 & 0
\end{array}\right), \\
& M_{1^{-+}}^{I=1}=M_{0}\left(\begin{array}{ll}
1 & 0 \\
0 & 1
\end{array}\right)+\kappa_{q Q}\left(\begin{array}{ll}
0 & 1 \\
1 & 0
\end{array}\right)-V_{L S}\left(\begin{array}{ll}
1 & 0 \\
0 & 1
\end{array}\right)+V_{0}\left(\begin{array}{cc}
-3 & 0 \\
0 & 1
\end{array}\right)+2 V_{T}\left(\begin{array}{ll}
1 & 0 \\
0 & 0
\end{array}\right),
\end{aligned}
$$




$$
\begin{aligned}
& M_{2^{-+}}^{I=0}=M_{0}\left(\begin{array}{ll}
1 & 0 \\
0 & 1
\end{array}\right)+\kappa_{q Q}\left(\begin{array}{ll}
0 & 1 \\
1 & 0
\end{array}\right)+V_{L S}\left(\begin{array}{ll}
1 & 0 \\
0 & 1
\end{array}\right)-3 V_{0}\left(\begin{array}{cc}
-3 & 0 \\
0 & 1
\end{array}\right)+\frac{6}{5} V_{T}\left(\begin{array}{ll}
1 & 0 \\
0 & 0
\end{array}\right) \\
& M_{2^{-+}}^{I=1}=M_{0}\left(\begin{array}{ll}
1 & 0 \\
0 & 1
\end{array}\right)+\kappa_{q Q}\left(\begin{array}{ll}
0 & 1 \\
1 & 0
\end{array}\right)+V_{L S}\left(\begin{array}{ll}
1 & 0 \\
0 & 1
\end{array}\right)+V_{0}\left(\begin{array}{cc}
-3 & 0 \\
0 & 1
\end{array}\right)-\frac{2}{5} V_{T}\left(\begin{array}{ll}
1 & 0 \\
0 & 0
\end{array}\right)
\end{aligned}
$$

\section{COMPARISON TO DATA}

The experimental evidence at the present time for a complete multiplet of $P=-$ hidden-charm 4-quark exotic states is fragmentary, as should be apparent from the overview of Sec. III. Only between 3-6 such states out of 28 in $\Sigma_{g}^{+}(1 P)$ have been clearly identified, most of which have $J^{P C}=1^{--}$. Moreover, according to Table I and Eqs. (21)-(22), this sector possesses a fourfold multiplicity for both $I=0$ and $I=1$, and therefore the exact $1^{--}$mass eigenvalues depend nontrivially upon the values of the 5 model parameters $M_{0}, \kappa_{q Q}, V_{L S}, V_{0}, V_{T}$ of the Hamiltonian in Eq. (20). In addition, if this model lacks an operatoreven one with a fairly small coefficient - that produces a pattern of mass matrix elements distinct from the pattern already present in Eqs. (21)-(22), then the effect on the spectrum of mass eigenvalues could be quite pronounced.

One could avoid such problems by constraining not the individual eigenvalues directly, but rather the coefficients of the characteristic polynomial of the $1^{--}$mass matrix $M_{1^{--}}$, such as its trace. However, obtaining constraints in this way requires one to be confident of which 4 states actually belong to the $1 P$ multiplet (more on this below). In addition, the factors of $\operatorname{Tr} M_{1^{--}}^{N}$ that comprise the characteristic polynomial are numerically dominated by $M_{0}(1 P)^{N}$ and are less sensitive to mass splittings. Furthermore, each factor of $\operatorname{Tr} M_{1^{--}}^{N}$ treats all eigenvalues symmetrically, while in practice one possesses indications from the data that a particular eigenvalue must be matched to a particular eigenvector representing a state with a particular pattern of decays as indicated by the quantum numbers of particles in the final state (again, more on this below).

As a result, the fits presented here are exploratory in nature, and depend crucially upon provisional assignments of observed states to particular roles in the model. Conversely, with so little data completely settled, this model provides definite predictions for the full spectrum of states, given any specific hypothesis for the identity of states thus far observed.

Even if a $1^{--}$state is experimentally confirmed, its membership in the $1 P$ multiplet can be in doubt. Specifically, $Y(4660)$ is well separated in mass from the other $1^{--}$states, and has been suggested as a $2 P$ state [23] or a $1 F$ state [30]. Using the methods of Ref. [23], which produce the values of $M_{0}(1 S)$ in Eq. (8) and $M_{0}(1 P)$ in Eq. (9), we find

$$
\begin{aligned}
& M_{0}(2 P)=4819-4824 \mathrm{MeV}, \\
& M_{0}(1 F)=4886-4891 \mathrm{MeV} .
\end{aligned}
$$

Since the $2 P-1 P$ splitting is then approximately $460 \mathrm{MeV}$, and the lightest $1 P$ candidate is $Y(4230)$ at $4218_{-4}^{+5} \mathrm{MeV}$, one may crudely estimate the lightest $2 P$ candidate to lie around $4680 \mathrm{MeV}$, which is not excessively higher than the measured value of $4643 \pm 9 \mathrm{MeV}$. The $1 F$ assignment, on the contrary, appears to be out of reach for $Y(4660)$. Even though $2 P$ quantum numbers certainly present a likely possibility, one may alternately consider (as is done below) a fit in which $Y(4660)$ is taken to be the highest of the $41 P$, $1^{--}$states.

If $Y(4660)$ is assumed to be an $I=0,1 P$ state, then one of $Y(4230), Y(4260), Y(4360), Y(4390)$ must be superfluous. As discussed in Sec. III, BESIII sees no strong evidence in the $J / \psi \pi \pi$ channel near $4260 \mathrm{MeV}$ of $Y(4260)$ (which interestingly, was historically the first of the $Y$ states observed [46]); rather, their measurement ascribed by the PDG to $Y(4260)$ agrees quite well with the value given for $Y(4230)$, and the latest BESIII value [39] agrees even better. One may then either suppose that all of the other $Y(4260)$ data, obtained via the process $e^{+} e^{-} \rightarrow \gamma Y$, represent a 4-quark state that is for some reason inaccessible to direct production via $e^{+} e^{-} \rightarrow Y$, or it is not an independent 4-quark state at all, being instead an obsolete experimental artifact properly subsumed into $Y(4230)$, or possibly even a signal of the low-lying $1^{--}$charmonium hybrid expected near that mass [29].

Recall also from Sec. III the interesting fact that the two BESIII measurements of $m_{Y(4360)}$ are very widely separated, with the higher mass being completely consistent with that of $Y(4390)$ (although its width differs by about $2 \sigma$ ), and the lower one near $4320 \mathrm{MeV}$ suggesting a new state, possibly subsuming what had been the high-mass tail of $Y(4260)$ events in other experiments. One may then consider a BESIII-only set of $1^{--}$states, given by $Y(4230)$, " $Y(4320)$," and $Y(4390)$, and then either predict a fourth $1^{--}$mass, or include $Y(4660)$ as the fourth $1^{--}$state in $1 P$.

The only other known $P=-$ candidate is the unconfirmed $Z_{c}(4240)$, whose $I=1, J^{P C}=0^{--}$quantum numbers are unique in the $1 P$ multiplet. Since its mass uncertainty (see Table II) is quite large, we choose to predict $m_{Z_{c}(4240)}$ from the fits rather than use it as an input.

Apart from the spectrum of mass eigenvalues, the most incisive observed feature of $1^{--}$states is that at least 2 of 
TABLE III. Results of fits to the Hamiltonian parameters $M_{0}, \kappa_{q Q}, V_{L S}, V_{0}, V_{T}$ in Eq. (20) using the $1^{--}$state assignments and masses summarized in the text as Cases 1-5. The mass of the $0^{--}, I=1$ state, to be compared with $m_{Z_{c}(4240)}$, is also predicted. All Hamiltonian parameters and mass predictions are given in units of MeV. Also presented are fractional amounts $P_{s_{Q \bar{Q}}=0}$ of the heavy-quark spin $s_{Q \bar{Q}}=0$ state in the mass eigenstates that correspond to $Y(4230)$ and $Y(4390)$.

\begin{tabular}{|c|c|c|c|c|c|c|c|c|c|c|c|c|c|}
\hline & $\chi_{\min }^{2} /$ d.o.f. & $M_{0}$ & $\kappa_{q Q}$ & $V_{L S}$ & $V_{0}$ & $V_{T}$ & \multicolumn{4}{|c|}{$M_{1^{--}}^{I=0}$} & $M_{0^{--}}^{I=1}$ & $P_{s_{Q \bar{Q}}=0}^{Y(4230)}$ & $P_{s_{Q \bar{Q}}=0}^{Y(439)}$ \\
\hline Case 1 & $0.457 / 4$ & 4356 & 12.7 & 61.6 & -14.7 & 7.0 & 4218.2 & 4230.4 & 4360.0 & 4393.9 & 4219.4 & 0.771 & 0.012 \\
\hline Case 2 & $2.47 / 5$ & 4355 & 19.9 & 59.8 & -12.9 & 8.3 & 4217.3 & 4237.9 & 4351.4 & 4393.3 & 4219.2 & 0.331 & 0.023 \\
\hline Case 3 & $15.2 / 6$ & 4357 & 44.6 & 42.9 & -1.8 & 5.5 & 4213.6 & 4262.9 & 4335.5 & 4394.8 & 4235.0 & 0.231 & 0.639 \\
\hline Case 4 & $5.33 / 6$ & 4357 & 43.7 & -53.7 & -0.2 & 10.4 & 4219.3 & 4374.5 & 4399.2 & 4637.6 & 4441.4 & 0.232 & 0.647 \\
\hline Case 5 & $3.76 / 6$ & 4356 & 43.2 & 49.0 & -2.7 & 3.8 & 4219.3 & 4257.1 & 4306.6 & 4385.7 & 4219.7 & 0.264 & 0.651 \\
\hline
\end{tabular}

them, $Y(4230)$ and $Y(4390)$, have been seen to decay to the $s_{Q \bar{Q}}=0$ charmonium state $h_{c}$. In addition, $Y(4230)$ is also seen to decay to the $s_{Q \bar{Q}}=1$ states $\chi_{c 0}$ and $\psi(2 S)$, indicating a significant mixing of $s_{Q \bar{Q}}=0$ and $s_{Q \bar{Q}}=1$ components within $Y(4230)$ (assuming that heavy-quark spin is conserved in the decays). The $Y(4390)$, on the other hand, has thus far only been seen to decay to $h_{c}$, although if the higher BESIII mass measurement $4384 \pm 4 \mathrm{MeV}$ for $e^{+} e^{-} \rightarrow \psi(2 S) \pi^{+} \pi^{-}$[40] ascribed to $Y(4360)$ actually belongs to $Y(4390)$, then this state also comprises a mixture of both $s_{Q \bar{Q}}$ components. But in any case, at least 2 of the $1^{--}$states have a significant coupling to $s_{Q \bar{Q}}=0$, while Table I indicates only 1 such state $\left(\tilde{X}_{0}^{(1)}\right)$ for each of $I=0$ and $I=1$. The $I=0 Y(4230)$ is clearly one of them, while $Y(4390)$ may be considered to carry either $I=0$ or $I=1$ (although again, recent BESIII $J / \psi \eta$ data [37] appear to eliminate the latter possibility). The probability $P_{s_{Q \bar{Q}}=0}$ of coupling to the $s_{Q \bar{Q}}=0$ component of a mass eigenstate is given by the square of the $\tilde{X}_{0}^{(1)}$ component of the normalized mass eigenvector.

We perform $\chi^{2}$ fits and compute $\chi_{\text {min }}^{2}$ for a given set of inputs $M_{0}, \kappa_{q Q}, V_{L S}, V_{0}, V_{T}$ by including terms for mass measurements and their uncertainties in the standard manner, while terms for $P_{s_{Q \bar{Q}}=0}$ are included in the form

$$
\Delta \chi^{2}=\left(\frac{\ln P_{s_{Q \bar{Q}}=0}-\ln f}{\epsilon}\right)^{2}
$$

so that $P_{s_{Q \bar{Q}}=0}$ is fit to a chosen branching fraction $f$, values $f \rightarrow 0$ thus being disfavored, and one unit of $\chi^{2}$ for this variable is approximately bracketed by the values $P_{s_{Q \bar{Q}}=0}=$ $f(1 \pm \epsilon)$. The exact branching fractions into $s_{Q \bar{Q}}=0$ and $s_{Q \bar{Q}}=1$ states are not yet known, so these constraints upon the $\chi^{2}$ function should be viewed as typical possible values rather than precise conditions imposed by the data. In particular, in light of the decay modes for $Y(4230)$ and $Y(4390)$ listed in Table II, some explicit examples we explore include fixing a substantial $s_{Q \bar{Q}=0}$ component of $f=\frac{1}{3}$ for $Y(4230)$ and a large $s_{Q \bar{Q}=0}$ component of $f=\frac{2}{3}$ for $Y(4390)$.
Having now motivated a diverse set of alternative ways to interpret the data for the $1^{--}$states, we now define a variety of specific scenarios to test against the model Hamiltonian of Eq. (20), using $\chi^{2}$ fits in the manner just outlined. Clearly, not every combination of possible interpretations of the data as described above is represented by these 5 cases, but they are useful in understanding how well various fits to Eq. (20) succeed in representing the full extant body of experimental results. The results of these fits are presented in Table III. Explicitly, the cases are

(1) $Y(4230), Y(4260), Y(4360), Y(4390)$ masses are as given in the PDG (Table II). No constraint is placed upon $P_{s_{Q \bar{Q}}=0}^{Y(4230)}$ or $P_{s_{Q \bar{Q}}=0}^{Y(4390)}$.

(2) $Y(4230), Y(4260), Y(4360), Y(4390)$ masses are as given in the PDG. $P_{s_{Q \bar{Q}}=0}^{Y(423)}$ is fit to $f=\frac{1}{3}$ with $\epsilon=0.1$, and $P_{s_{Q \bar{Q}}=0}^{Y(4390)}$ is unconstrained.

(3) $Y(4230), Y(4360)$, and $Y(4390)$ masses are as given in the PDG, while $m_{Y(4260)}=4251 \pm 6 \mathrm{MeV}$, which is the weighted average of the 3 PDG values not including the low BESIII value [38]. $P_{s_{Q \bar{Q}}=0}^{Y(4230)}$ is fit to $f=\frac{1}{3}$ with $\epsilon=0.2$, and $P_{s_{Q \bar{Q}}=0}^{Y(4390)}$ is fit to $f=\frac{2}{3}$ with $\epsilon=0.05$.

(4) $Y(4360), Y(4390)$, and $Y(4660)$ masses are as given in the PDG, but $Y(4260)$ is assumed not to exist, and $m_{Y(4230)}=4220.1 \pm 2.9 \mathrm{MeV}$ is the weighted average of the PDG values combined with the newer BESIII measurements [36,39]. $P_{s_{Q \bar{Q}}=0}$ values are as given in Case 3.

(5) $m_{Y(4230)}$ is as given in Case $4 ; m_{Y(4260)}$ is as given in Case 3; $m$ " $Y(4320) "=4320 \pm 13 \mathrm{MeV}$ is the lower BESIII $Y(4360)$ mass measurement from [38]; $m_{Y(4390)}=4386 \pm 4 \mathrm{MeV}$ is the weighted average of the PDG value and the upper BESIII mass measurement from [40]. $P_{s_{Q \bar{Q}}=0}$ values are as given in Case 3.

The robustness of the $\chi_{\min }^{2}$ values are checked by selecting several initial sets of parameters and confirming that the same minimum is reached for each such choice. Neither uncertainties on the output parameters nor a 
covariance matrix are presented for the results of Table III, partly since the $1^{--}$mass inputs are eigenvalues of a $4 \times 4$ matrix, which satisfy a highly nonlinear characteristic equation. More broadly, however, presenting such detailed fits to data that remain strongly in flux would suggest more confidence in the exact fit values of the output parameters than is warranted at this time.

The first feature of note in Table III is that the values of $\chi_{\min }^{2}$ are entirely satisfactory for Cases $1,2,4$, and 5, and large for Case 3 . These results do not simply mean that some assignments of states are intrinsically better than others; rather, they indicate which pieces of data and which parameters are instrumental in driving quality of the fit, as discussed below.

The fit values of $M_{0}(1 P)$ are remarkably robust, even in Case 4, which treats $Y(4660)$ as the highest $1 P$ state. Note that the $M_{0}(1 P)$ value is not taken from Eq. (9), but rather emerges via the $\chi^{2}$ optimization. The price of treating $Y(4660)$ as a $1 P$ rather than a $2 P$ state is the prediction that the sole $I=1,0^{--}$state in the $1 P$ multiplet is much heavier than the observed $Z_{c}(4240)$, while all of the other fits provide satisfactory values for $m_{Z_{c}(4240)}$. The value of $V_{L S}$ is also rather stable in all cases that do not include $Y(4660)$; $V_{L S}$ appears to be the primary parameter responsible for the largest mass splittings within the $1 P$ multiplet.

If $Y(4660)$ is instead required to be a $2 P$ state, then one may begin with the minimal assumption in Case 1 that the 4 $1 P Y$ states are just the lighter $1^{--}$ones listed in the PDG, and the mixings $P_{s_{Q \bar{Q}}=0}$ are allowed to vary freely. One finds, unsurprisingly, a perfect fit to the masses, and that almost all of the $s_{Q \bar{Q}}=0$ strength resides with $Y(4230)$, but almost none with $Y(4390)$, contrary to observation. The value of $\kappa_{q Q}$ obtained actually turns out to be smaller than the value obtained in Eq. (8) for the $1 S$ multiplet.

However, when one also fits to $P_{s_{Q \bar{Q}}=0}^{Y(4230)}=\frac{1}{3}$ [Case 2], the $\chi_{\min }^{2} /$ d.o.f. rises somewhat - still providing a good fit—but most significantly, $\kappa_{q Q}$ becomes almost equal to the value in Eq. (8). If one then also attempts to fit to $P_{s_{Q \bar{Q}}=0}^{Y(4230)}=\frac{2}{3}$ as well (a case not presented in Table III), then the value of $\chi_{\min }^{2} /$ d.o.f. rises dramatically, its increase driven by the tendency of the fit to prefer a larger $m_{Y(4260)}$ and a smaller $m_{Y(4360)}$ than the PDG values.

Case 3 represents a halfway point designed to relieve this tension; $m_{Y(4260)}$ is taken to be the average obtained from the larger, non-BESIII PDG measurements, with the smaller BESIII measurement subsumed into $m_{Y(4230)}$. One finds the resulting value of $\kappa_{q Q}$ to be much larger, a feature apparently necessary to provide substantial $P_{s_{Q \bar{Q}}=0}$ values for both $Y(4230)$ and $Y(4390)$, and the larger $\chi_{\min }^{2} /$ d.o.f. value is driven primarily by the preference of the fit for a smaller $m_{Y(4360)}$.

We therefore modify Case 3 to consider in Case 5 the interesting possibility discussed above (inspired by BESIII measurements) that the $Y(4360)$ data actually represent a combination of data that belongs to $Y(4390)$ and a lighter state " $Y(4320)$ ". One then obtains an excellent fit to all observables. The stable values of $M_{0}(1 P), V_{L S}$, and large value of $\kappa_{q Q}$ have already been noted. In addition, most of the fits in Table III prefer small, generally negative values of $V_{0}$, quite different from the $1 S$ value in Eq. (8), suggesting a rather different isospin-dependent interaction in the $1 P$ states. Still, one must note that the $V_{0}$ and $V_{T}$ couplings in the Hamiltonian of Eq. (20) solely couple to static light quarks; the presence of nonzero orbital angular momentum clearly changes the physical interpretation one should apply to the corresponding operators.

We do not insist that this particular choice of masses represents the true spectrum of $I=0,1^{--} 1 P$ states that will eventually be clarified by future experiments; in particular, our simplistic estimates of $P_{s_{Q \bar{Q}}=0}$ values certainly skew the fit results, and may not bear up under future scrutiny. Nevertheless, our purpose here is to show the versatility of the Hamiltonian of Eq. (20) in accommodating data sets not unlike those already obtained, with the key restriction of the dynamical diquark model being that exactly $4 I=0,1^{--}$states and $1 I=1,0^{--}$state occur in the $1 P$ multiplet.

Using the fit values obtained for the parameters $M_{0}, \kappa_{q Q}$, $V_{L S}, V_{0}, V_{T}$ from any of the cases listed in Table III, one may predict the masses for all 28 isomultiplets within the $1 P$ multiplet. For concreteness, we use the inputs of Case 5, and present these results in Table IV, with a corresponding level diagram in Fig. 1 . The $I=0,1^{--}$states (called $Y$ above, and labeled as $\psi$ by the PDG, see Table I) all fit well with set of the mass observations specified in Case 5; as noted above, this part of the fit can be successfully realized by any of the given cases. In addition, the predicted mass of the $I=1,0^{--}$state nicely agrees with the measured mass of $Z_{c}(4240)$, which has precisely these quantum numbers.

The $I=1,1^{--}$states (PDG designation: $R_{c 1}$ ) are particularly interesting, as the subset with $I_{3}=0$ should

TABLE IV. Prediction of the 28 isomultiplet masses (in MeV) of the $\Sigma_{g}^{+}(1 P)$ multiplet, using the Hamiltonian of Eq. (20) and the numerical values of the parameters obtained from fit to the state assignment of Case 5 as given in Table III. Boldface indicates fit outputs for states whose measured masses are used as inputs for the fit.

\begin{tabular}{lllll}
\hline \hline$J^{P C}$ & \multicolumn{2}{c}{$I=0$} & \multicolumn{2}{c}{$I=1$} \\
\hline $1^{--}$ & $\mathbf{4 2 1 9 . 3}$ & $\mathbf{4 2 5 7 . 1}$ & 4224.4 & 4241.6 \\
& $\mathbf{4 3 0 6 . 6}$ & $\mathbf{4 3 8 5 . 7}$ & 4261.9 & 4404.7 \\
$0^{--}$ & 4200.3 & & 4219.7 & \\
$2^{--}$ & 4337.8 & 4372.2 & 4351.0 & 4361.6 \\
$3^{--}$ & 4509.7 & & 4493.0 & \\
$0^{-+}$ & 4228.8 & 4316.2 & 4209.9 & 4296.4 \\
$1^{-+}$ & 4236.7 & 4338.8 & 4269.3 & 4357.5 \\
$2^{-+}$ & 4354.0 & 4444.6 & 4363.5 & 4450.3 \\
\hline \hline
\end{tabular}




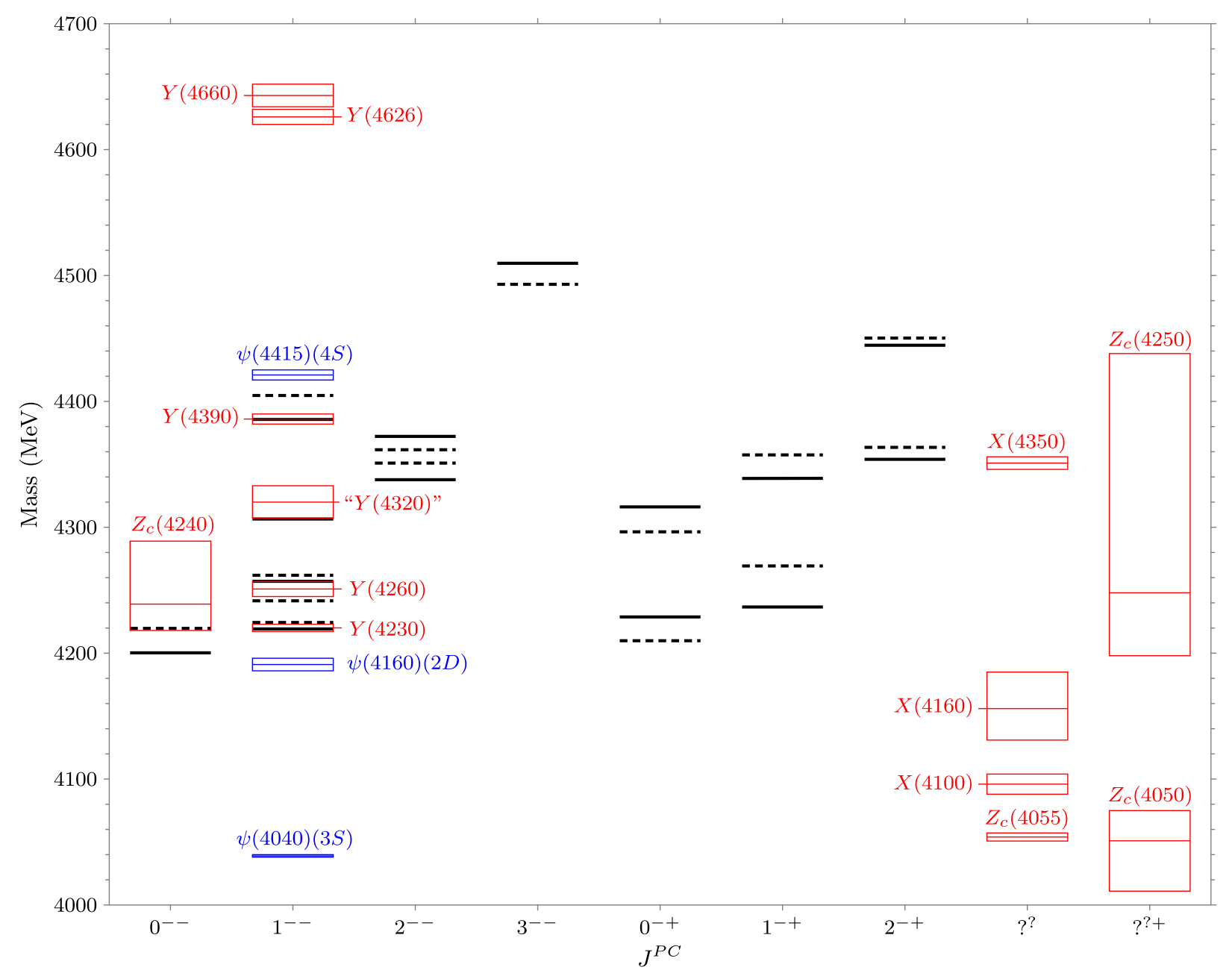

FIG. 1. Level diagram for the 28 negative-parity states of the $\Sigma_{g}^{+}(1 P)$ multiplet, with masses as given in Table IV and predicted using the $Y$-state assignment of Case 5. Heavy solid (dashed) lines indicate $I=0(I=1)$ states, respectively. Each observed state mass, including its central value and uncertainty, is presented as a rectangle, with exotic candidates in red and conventional charmonium states in blue. Observed states labeled $Z_{c}$ are all $I=1$, and all others are $I=0$. The final two columns present observed states for which not all $J^{P C}$ values are known, although in addition, $Z_{c}(4055)$ is $C=-$ and $X(4350)$ is $C=+$.

also be produced in $e^{+} e^{-}$collisions, and as noted above, might be mistaken for $Y$ states. Remarkably, in Table IV and Fig. 1 all such $I=1$ states lie fairly close to ones with $I=0$ (and not necessarily in a one-to-one fashion). The signal of the highest one, at $4405 \mathrm{MeV}$, might even be obscured by the conventional charmonium state $\psi(4415)$. Part of the confusion about $1^{--}$resonance data might thus ultimately find its origin in overlapping signals from states with distinct isospin eigenvalues, which would only be completely separated either through observing nearlydegenerate charged partners for the $I=1$ states, or by discerning final states consisting of conventional charmonium plus a light hadron state of a known isospin eigenvalue.

Beyond this point, we have already noted that no other observed states have a confirmed $P=-$ eigenvalue, excepting the $Y(4626)$ (which we have argued to be a $c \bar{c} s \bar{s}$ state) and $Y(4660)$ (which, in Case 5 , is a $2 P$ state). The lowest predicted mass in Table IV and Fig. 1 is that of the $I=0,0^{--}$state at $4200 \mathrm{MeV}$, for which $X(4160)$ (currently possessing a very large mass uncertainty and completely unknown quantum numbers) is at least a plausible candidate. Outside of the $1^{--}$sector, one finds that all states of $I=1, J^{P C}=(0,1,2)^{-+}$lie between 4210 and $4450 \mathrm{MeV}$, an enormous range that amusingly almost exactly matches the mass range of the unconfirmed, $C=+Z_{c}(4250)$; very possibly, the $Z_{c}(4250)$ observation could amount to the overlapping effect of several of these states. Lastly, the unconfirmed $I=0 X(4350)$ has been suggested as a $c \bar{c} s \bar{s}$ state due to its $J / \psi \phi$ decay mode [34], but its mass and $C=+$ eigenvalue also make it a candidate $2^{-+}$state.

\section{CONCLUSIONS}

In this paper we have studied the lightest hidden-charm negative-parity exotic meson candidates. In diquark models (specifically, the dynamical diquark model) in which these 
states consist of two separated diquarks $(c q)\left(\bar{c} \bar{q}^{\prime}\right)$, one may classify the states according to Born-Oppenheimer approximation quantum numbers. Then the lowest multiplets (as indicated by lattice QCD simulations of the glue field connecting the diquark pair) are $\Sigma_{g}^{+}(1 S)$ and $\Sigma_{g}^{+}(1 P)$, and all states within these multiplets carry parity + and - , respectively. The 12 isomultiplets of $\Sigma_{g}^{+}(1 S)$ were studied previously by establishing a 3-parameter Hamiltonian that respected all known phenomenology of the low-lying $P=+$ states such as $X(3872)$. The current paper extends this analysis to the 28 isomultiplets of $\Sigma_{g}^{+}(1 P)$ by introducing 2 new parameters into the Hamiltonian, which represent spin-orbit and isospin-dependent tensor terms.

The current experimental status of these $P=-$ states remains in flux, notably in the $J^{P C}=1^{--}$sector. For example, even the famous $Y(4260)$ — known for 15 yearsmight actually be a combination of other states like $Y(4230)$, or even a charmonium hybrid. Consequently, we have examined a variety of hypotheses for identifying the $4 I=0, J^{P C}=1^{--}$diquark-model $1 P$ states with observed resonances. An additional and rather restrictive constraint appears upon noticing that only 1 linear combination of these 4 states couples to charmonium in a heavyquark spin singlet ( i.e., $h_{c}$ rather than $\psi$ or $\chi_{c}$ ), while at least 2 of the observed states [Y(4230) and $Y(4390)]$ have significant decays to $h_{c}$. Nevertheless, scenarios can be found in which all current phenomenological constraints are satisfied in a detailed fit. In this regard, we have discussed which particular pieces of data are the most significant ones in allowing or impeding successful fits.

One remarkable result of the fits is the robustness of the prediction of the $1 P$ multiplet average mass $(\approx 4358 \mathrm{MeV})$ using only data from the $1^{--}$sector, which agrees extremely well with the result of combining the $1 S$ value obtained from previous work with a calculation of the $1 S-1 P$ splitting using the aforementioned lattice glue simulations. The dynamical diquark model appears to be fully self-consistent in this important regard.

Once the 5 parameters of the Hamiltonian are determined numerically, then the entire spectrum of 28 isomultiplets is predicted. Among the results obtained, we note that the masses of the known $I=0,1^{--} Y$ states can easily be fitted in a variety of scenarios. Somewhat more discriminating is the constraint that the $Y(4230)$ and $Y(4390)$ have both been observed to decay to $h_{c}$, but excellent fits satisfying this additional criterion have also been achieved. Supposing that $Y(4660)$ is a $2 P$ state, the mass prediction of the sole $I=1,0^{--}$state matches that of the observed state $Z_{c}(4240)$ that has these quantum numbers.

Future experiments will undoubtedly resolve the ambiguities of spectroscopy and decay patterns discussed here, making a comparison of the model to data much more straightforward. New states may emerge and old ones may be de-established, or be identified as hybrids rather than as 4-quark states. Other Hamiltonian operators essential for providing important splittings may turn out to have been neglected in this first attempt, or indeed, the diquark model itself may turn out to have some fatal flaw. But even in that worst-case scenario, the operators used here still have physical significance in a generic 4-quark model, and many more 4-quark states are still predicted to exist and remain to be discovered.

\section{ACKNOWLEDGMENTS}

The authors are grateful to R. E. Mitchell for important insights into the current status of the $1^{--}$sector. This work was supported by the National Science Foundation (NSF) under Grant No. PHY-1803912.

\section{APPENDIX: EVALUATION OF THE TENSOR OPERATOR}

The computation of matrix elements of the tensor operator

$$
S_{12} \equiv 3 \boldsymbol{\sigma}_{1} \cdot \boldsymbol{r} \boldsymbol{\sigma}_{2} \cdot \boldsymbol{r} / r^{2}-\boldsymbol{\sigma}_{1} \cdot \boldsymbol{\sigma}_{2},
$$

where $\sigma$ here denotes not just spin- $-\frac{1}{2}$ Pauli matrices, but more generally twice the canonically normalized spin generators $\mathbf{S}$ for arbitrary spin $s$, is given in many references, e.g., Ref. [45]:

$$
\left\langle L^{\prime}, S^{\prime}, J\left|S_{12}\right| L, S, J\right\rangle=(-1)^{S+J} \sqrt{30[L]\left[L^{\prime}\right][S]\left[S^{\prime}\right]}\left\{\begin{array}{lll}
J & S^{\prime} & L^{\prime} \\
2 & L & S
\end{array}\right\}\left\{\begin{array}{ccc}
L^{\prime} & 2 & L \\
0 & 0 & 0
\end{array}\right\}\left\{\begin{array}{lll}
s_{1} & s_{2} & S \\
s_{3} & s_{4} & S^{\prime} \\
1 & 1 & 2
\end{array}\right\}\left\langle s_{1}\left\|\boldsymbol{\sigma}_{1}\right\| s_{3}\right\rangle\left\langle s_{2}\left\|\boldsymbol{\sigma}_{2}\right\| s_{4}\right\rangle
$$

where again, $[j] \equiv 2 j+1$. The reduced matrix elements of the angular momentum generators are given by

$$
\left\langle j^{\prime}|| \mathbf{j}|| j\right\rangle=\sqrt{j(2 j+1)(j+1)} \delta_{j^{\prime} j} .
$$

Using Eq. (A3) to simplify Eq. (A2) and applying the many restrictions on allowed values of angular momentum that follow, one obtains Eq. (17).
Section IV tabulates the matrix elements of $S_{12}^{(q \bar{q})}$, i.e., the tensor operator for which the individual spin operators in Eq. (A1) and the total spins $S, S^{\prime}$ in Eq. (A2) refer to the light-quark pair $q \bar{q}$ alone. In this Appendix we present the results for $S_{12}^{(\delta \bar{\delta})}$, the tensor operator for which the individual spin operators in Eq. (A1) and the total spins $S, S^{\prime}$ in Eq. (A2) refer to the diquark pair $\delta, \bar{\delta}$. The calculation of 
$S_{12}^{(\delta \bar{\delta})}$ matrix elements is actually somewhat simpler than the matrix elements of $S_{12}^{(q \bar{q})}$, since the total diquark spin $s_{\delta \bar{\delta}}$ to be used in Eq. (A2) as $S$ or $S^{\prime}$ is just the total quark spin $S$, which is a good quantum number for the $P$-wave states listed in Table I. Thus, a recoupling such as that in Eq. (19) is not needed.

We now tabulate matrix elements $\Delta M$ of the diquark spin-dependent operators in Eqs. (7) and (16) for the $1 P$ states:

$$
\Delta H=\boldsymbol{\tau}_{q} \cdot \boldsymbol{\tau}_{\bar{q}}\left(V_{1} \boldsymbol{\sigma}_{\delta} \cdot \boldsymbol{\sigma}_{\bar{\delta}}+V_{T}^{\prime} S_{12}^{(\delta \bar{\delta})}\right),
$$

where the isospin-dependent part $\boldsymbol{\tau}_{q} \cdot \boldsymbol{\tau}_{\bar{q}}$ simply provides a factor of $\{-3,+1\}$ for $I=\{0,1\}$, respectively. The results, collected by $J^{P C}$ eigenvalues, are given in the same order as appearing in Table I and in Eqs. (21)(34). However, the matrix elements for the isospinindependent terms of Eq. (20) $\left(M_{0}, \kappa_{q Q}, V_{L S}\right)$ remain the same.

$$
\begin{aligned}
& \Delta M_{1^{--}}^{I=0}=-6 V_{1}\left(\begin{array}{cccc}
-3 & \sqrt{3} & 0 & 0 \\
\sqrt{3} & -1 & 0 & 0 \\
0 & 0 & 0 & 0 \\
0 & 0 & 0 & 2
\end{array}\right)-12 V_{T}^{\prime}\left(\begin{array}{cccc}
0 & 0 & 0 & \sqrt{\frac{3}{5}} \\
0 & 0 & 0 & -\frac{1}{\sqrt{5}} \\
0 & 0 & 0 & 0 \\
\sqrt{\frac{3}{5}} & -\frac{1}{\sqrt{5}} & 0 & -\frac{7}{5}
\end{array}\right), \\
& \Delta M_{1}^{I=1}=2 V_{1}\left(\begin{array}{cccc}
-3 & \sqrt{3} & 0 & 0 \\
\sqrt{3} & -1 & 0 & 0 \\
0 & 0 & 0 & 0 \\
0 & 0 & 0 & 2
\end{array}\right)+4 V_{T}^{\prime}\left(\begin{array}{cccc}
0 & 0 & 0 & \sqrt{\frac{3}{5}} \\
0 & 0 & 0 & -\frac{1}{\sqrt{5}} \\
0 & 0 & 0 & 0 \\
\sqrt{\frac{3}{5}} & -\frac{1}{\sqrt{5}} & 0 & -\frac{7}{5}
\end{array}\right), \\
& \Delta M_{0^{--}}^{I=0}=0, \\
& \Delta M_{0^{--}}^{I=1}=0, \\
& \Delta M_{2^{--}}^{I=0}=-12\left(V_{1}+\frac{7}{5} V_{T}^{\prime}\right)\left(\begin{array}{ll}
0 & 0 \\
0 & 1
\end{array}\right), \\
& \Delta M_{2^{--}}^{I=1}=4\left(V_{1}+\frac{7}{5} V_{T}^{\prime}\right)\left(\begin{array}{ll}
0 & 0 \\
0 & 1
\end{array}\right), \\
& \Delta M_{3^{--}}^{I=0}=-12\left(V_{1}-\frac{2}{5} V_{T}^{\prime}\right), \\
& \Delta M_{3^{--}}^{I=1}=4\left(V_{1}-\frac{2}{5} V_{T}^{\prime}\right), \\
& \Delta M_{0^{-+}}^{I=0}=6\left(V_{1}+2 V_{T}^{\prime}\right)\left(\begin{array}{ll}
1 & 1 \\
1 & 1
\end{array}\right), \\
& \Delta M_{0^{-+}}^{I=1}=-2\left(V_{1}+2 V_{T}^{\prime}\right)\left(\begin{array}{ll}
1 & 1 \\
1 & 1
\end{array}\right), \\
& \Delta M_{1^{-+}}^{I=0}=6\left(V_{1}-V_{T}^{\prime}\right)\left(\begin{array}{ll}
1 & 1 \\
1 & 1
\end{array}\right), \\
& \Delta M_{1^{-+}}^{I=1}=-2\left(V_{1}-V_{T}^{\prime}\right)\left(\begin{array}{ll}
1 & 1 \\
1 & 1
\end{array}\right), \\
& \Delta M_{2^{-+}}^{I=0}=6\left(V_{1}+\frac{1}{5} V_{T}^{\prime}\right)\left(\begin{array}{ll}
1 & 1 \\
1 & 1
\end{array}\right), \\
& \Delta M_{2^{-+}}^{I=1}=-2\left(V_{1}+\frac{1}{5} V_{T}^{\prime}\right)\left(\begin{array}{ll}
1 & 1 \\
1 & 1
\end{array}\right) \text {. }
\end{aligned}
$$


[1] R. Lebed, R. Mitchell, and E. Swanson, Prog. Part. Nucl. Phys. 93, 143 (2017).

[2] H.-X. Chen, W. Chen, X. Liu, and S.-L. Zhu, Phys. Rep. 639, 1 (2016).

[3] A. Hosaka, T. Iijima, K. Miyabayashi, Y. Sakai, and S. Yasui, Prog. Theor. Exp. Phys. 2016, 062 C01 (2016).

[4] A. Esposito, A. Pilloni, and A. Polosa, Phys. Rep. 668, 1 (2017).

[5] F.-K. Guo, C. Hanhart, U.-G. Meißner, Q. Wang, Q. Zhao, and B.-S. Zou, Rev. Mod. Phys. 90, 015004 (2018).

[6] A. Ali, J. Lange, and S. Stone, Prog. Part. Nucl. Phys. 97, 123 (2017).

[7] S. Olsen, T. Skwarnicki, and D. Zieminska, Rev. Mod. Phys. 90, 015003 (2018).

[8] M. Karliner, J. Rosner, and T. Skwarnicki, Annu. Rev. Nucl. Part. Sci. 68, 17 (2018).

[9] C.-Z. Yuan, Int. J. Mod. Phys. A 33, 1830018 (2018).

[10] Y.-R. Liu, H.-X. Chen, W. Chen, X. Liu, and S.-L. Zhu, Prog. Part. Nucl. Phys. 107, 237 (2019).

[11] N. Brambilla, S. Eidelman, C. Hanhart, A. Nefediev, C.-P. Shen, C. Thomas, A. Vairo, and C.-Z. Yuan, arXiv: 1907.07583.

[12] S. Brodsky, D. Hwang, and R. Lebed, Phys. Rev. Lett. 113, 112001 (2014).

[13] R. Lebed, Phys. Rev. D 96, 116003 (2017).

[14] R. Lebed, Phys. Lett. B 749, 454 (2015).

[15] M. Karliner and H. Lipkin, Phys. Lett. B 638, 221 (2006).

[16] A. Ali, I. Ahmed, M. Aslam, A. Ya. Parkhomenko, and A. Rehman, J. High Energy Phys. 10 (2019) 256.

[17] L. Griffiths, C. Michael, and P. Rakow, Phys. Lett. 129B, 351 (1983).

[18] K. Juge, J. Kuti, and C. Morningstar, Nucl. Phys. B, Proc. Suppl. 63, 326 (1998).

[19] K. Juge, J. Kuti, and C. Morningstar, Phys. Rev. Lett. 82, 4400 (1999).

[20] K. Juge, J. Kuti, and C. Morningstar, Phys. Rev. Lett. 90, 161601 (2003).

[21] S. Capitani, O. Philipsen, C. Reisinger, C. Riehl, and M. Wagner, Phys. Rev. D 99, 034502 (2019).

[22] G. Bali and A. Pineda, Phys. Rev. D 69, 094001 (2004).

[23] J. Giron, R. Lebed, and C. Peterson, J. High Energy Phys. 05 (2019) 061.
[24] J. Giron, R. Lebed, and C. Peterson, J. High Energy Phys. 01 (2020) 124.

[25] J. Giron and R. Lebed (to be published).

[26] Z.-G. Wang, Eur. Phys. J. C 78, 933 (2018).

[27] Z.-G. Wang, Eur. Phys. J. C 79, 29 (2019).

[28] M. Tanabashi and et al. (Particle Data Group), Phys. Rev. D 98, 030001 (2018).

[29] L. Liu, G. Moir, M. Peardon, S. Ryan, C. Thomas, P. Vilaseca, J. Dudek, R. Edwards, B. Joo, and D. Richards (Hadron Spectrum Collaboration), J. High Energy Phys. 07 (2012) 126.

[30] L. Maiani, F. Piccinini, A. Polosa, and V. Riquer, Phys. Rev. D 89, 114010 (2014).

[31] M. Cleven, F.-K. Guo, C. Hanhart, Q. Wang, and Q. Zhao, Phys. Rev. D 92, 014005 (2015).

[32] S. Jia et al. (Belle Collaboration), Phys. Rev. D 100, 111103 (2019).

[33] S. Jia et al. (Belle Collaboration), arXiv:2004.02404.

[34] R. Lebed and A. Polosa, Phys. Rev. D 93, 094024 (2016).

[35] R. Aaij et al. (LHCb Collaboration), Phys. Rev. Lett. 112, 222002 (2014).

[36] M. Ablikim et al. (BESIII Collaboration), Phys. Rev. Lett. 122, 102002 (2019).

[37] M. Ablikim et al. (BESIII Collaboration), arXiv: 2003.03705.

[38] M. Ablikim and et al. (BESIII Collaboration), Phys. Rev. Lett. 118, 092001 (2017).

[39] M. Ablikim et al. (BESIII Collaboration), Phys. Rev. D 99, 091103 (2019).

[40] M. Ablikim et al. (BESIII Collaboration), Phys. Rev. D 96, 032004 (2017); 99, 019903(E) (2019).

[41] R. Aaij and et al. (LHCb Collaboration), Phys. Rev. Lett. 122, 152002 (2019).

[42] C.-Z. Yuan et al. (Belle Collaboration), Phys. Rev. Lett. 99, 182004 (2007).

[43] M. Ablikim et al. (BESIII Collaboration), Phys. Rev. D 97, 052001 (2018).

[44] S.-K. Choi et al. (Belle Collaboration), Phys. Rev. D 84, 052004 (2011).

[45] A. de Shalit and I. Talmi, Nuclear Shell Theory, Pure and Applied Physics (Academic Press, New York, 1963).

[46] B. Aubert et al. (BABAR Collaboration), Phys. Rev. Lett. 95, 142001 (2005). 